Revue internationale P.M.E.

Économie et gestion de la petite et moyenne entreprise

Revure

internationale

PME

\title{
Les PME à forte croissance en Italie : succès étonnants et désagréables surprises
}

\section{Alberto Bramanti}

Volume 14, numéro 3-4, 2001

URI : https://id.erudit.org/iderudit/1008699ar

DOI : https://doi.org/10.7202/1008699ar

Aller au sommaire du numéro

\section{Éditeur(s)}

Presses de l’Université du Québec

ISSN

0776-5436 (imprimé)

1918-9699 (numérique)

Découvrir la revue

Citer cet article

Bramanti, A. (2001). Les PME à forte croissance en Italie : succès étonnants et désagréables surprises. Revue internationale P.M.E., 14(3-4), 91-127.

https://doi.org/10.7202/1008699ar
Résumé de l'article

Le présent travail précise le rôle que les PME assument dans la création de nouveaux emplois en Italie. En même temps, il reconstruit, en les interprétant, les parcours qui ont été suivis par le sous-ensemble des PME qui ont crû le plus dans la première moitié des années 1990.

Dans la partie empirique originale, qui a été menée sur un échantillon de 70 entreprises manufacturières à forte croissance, on fait une distinction entre quatre groupes d'entreprises relativement homogènes : les jeunes, les exportatrices, les dominées et celles qui appartiennent à un groupe.

Le croisement de ces quatre groupes avec les trois types facteurs de croissance, externes, internes et les motivations personnelles du dirigeant, nous permet d'identifier et d'interpréter la multiplicité des trajectoires ainsi que l'articulation des différentes combinaisons de facteurs. Un des éléments communs qui les distinguent est que les PME sont fortement dominées par l'entrepreneur. Il s'agit de PME qui sont compétitives sur leurs marchés et assez innovatrices. Ces PME se concentrent sur la différenciation et sur les services. Elles sont habituées de recourir à des stratégies à moyen et long terme, tout en planifiant les phases de croissance.
Ce document est protégé par la loi sur le droit d'auteur. L'utilisation des services d'Érudit (y compris la reproduction) est assujettie à sa politique d'utilisation que vous pouvez consulter en ligne.

https://apropos.erudit.org/fr/usagers/politique-dutilisation/ 


\title{
Les PME à forte croissance en Italie: succès étonnants et désagréables surprises
}

Alberto BRAMANTI ${ }^{1}$

Istituto di Economia Politica

Università Luigi-Bocconi

MOTS CLÉS

PME - Facteurs de croissance - Politiques

\begin{abstract}
RÉSUMÉ
Le présent travail précise le rôle que les PME assument dans la création de nouveaux emplois en Italie. En même temps, il reconstruit, en les interprétant, les parcours qui ont été suivis par le sous-ensemble des PME qui ont crû le plus dans la première moitié des années 1990.
\end{abstract}

Dans la partie empirique originale, qui a été menée sur un échantillon de 70 entreprises manufacturières à forte croissance, on fait une distinction entre quatre groupes d'entreprises relativement homogènes: les jeunes, les exportatrices, les dominées et celles qui appartiennent à un groupe.

Le croisement de ces quatre groupes avec les trois types facteurs de croissance, externes, internes et les motivations personnelles du dirigeant, nous permet d'identifier et d'interpréter la multiplicité des trajectoires ainsi que l'articulation des différentes combinaisons de facteurs. Un des éléments communs qui les distinguent

\section{L'AUTEUR}

Alberto Bramantı est professeur associé en économie appliquée à l'Université Bocconi à Milan. II travaille dans le domaine de l'économie régionale et s'occupe en particulier de l'évolution dynamique des PME et des politiques de développement. II a été membre du conseil de l'Association italienne des sciences régionales. Adresse : Via U. Gobbi, 5-20136 Milan. Courriel <alberto.bramanti@uni-bocconi.it>.

1. Je voudrais remercier Massimiliano Riggi et Massimo Zucca pour le précieux travail qu'ils ont fait dans la gestion de base de données et dans l'analyse des données. Je voudrais aussi remercier Michele Scarpinato pour avoir travaillé avec moi à tout le projet de recherche de l'OCDE. 
est que les PME sont fortement dominées par l'entrepreneur. II s'agit de PME qui sont compétitives sur leurs marchés et assez innovatrices. Ces PME se concentrent sur la différenciation et sur les services. Elles sont habituées de recourir à des stratégies à moyen et long terme, tout en planifiant les phases de croissance.

\begin{abstract}
The study is devoted to SMFs and their role in creating net jobs in Italy. At the same time, it presents and discusses the development paths followed by those firms, which shared the highest growth performance in the first half of the 1990s.

Within an original field research - carried out with a sample of 70 industrial high-growth firms - we distinguish among four different clusters: the young, the exporters, firms belonging to a group and subordinate firms.

The study goes on crossing these four groups with three different factors external, internal and motivational - and, in doing so, it is possible to identify and decode the multiplicity of growth paths and the different factors mix.

The common elements, which are to some extent present, show a SMF strongly directed by the entrepreneur, a firm competitive on its own markets, quite innovative, very attentive to customisation and service, accustomed to articulate a medium-long term strategy, able to programme its own steps on the growth path.
\end{abstract}

\title{
RESUMEN
}

El presente trabajo clarifica la importancia que tienen las PIMES en la creación de nuevos puestos de trabajo en Italia. En mismo tiempo reconstruye, también en interpretándolo, el camino que ha sido seguido por el subconjunto de las PIMES que aumentó lo más en la primera mitad de los años noventa.

En la parte empírica original, que fue realizada a base de una entrevista con 70 compañías de fabricación de grande crecimiento, se hizo una distinción entre cuatro grupos de compañías, todos homogenos en su interior: las jóvenes, las empresas de exportación, las "dominadas» y aquellas que apartenecen a un grupo.

El conjunto de estos cuatro grupos con los tres differentes factores de crecimiento los exteriores, los interiores y los aspectos motivantes nos permete de identificar y también de interpretar la multiplicidad de cursos de las empresas y de articular las differentes mezclas de factores. No obstante, también hay elementos communes que se distingen, mostrandonos des PIMES que son fuertemente dominadas por los empresarios. Se trata des PIMES que son competitivas en sus mercados y que también son bastante innovativas. Eses PIMES se concetran fuertemente en la personalización y en los servicios. Ellos son acostumbradas a formular estrategías de medio a largo periodo, planificando sus fases de crecimiento.

\section{ZUSAMMENFASSUNG}

Die vorliegende Arbeit verdeutlicht die Rolle der KMU in Italien in bezug auf die Gründung von neuen Arbeitsplätzen. Gleichzeitig werden die Entwicklungswege aufgezeigt, die von denjenigen KMU verfolgt wurden, die am meistens in das Wachstum der ersten Hälfte der 90er Jahre geglaubt hatten. 
Im empirischen Teil, der auf einer Stichprobe von 70 stark wachsenden Produktionsunternehmen aufbaut, wurde eine Unterscheidung zwischen vier relativ homogenen Unternehmengruppen vorgenommen : die Jungen, die Exportorientierten, die Dominanten und diejenigen die zu einer Gruppe gehören.

Die Kreuzung/Mischung von diesen vier Gruppen mit den drei verschiedenen Wachstumsfaktoren (Wachstumsfaktoren aufgrund der Umwelt, aufgrund interner und Motivationsgründe) erlauben uns, die Vielzahl der Entwicklungswege zu identifizieren und zu interpretieren. Ein gemeinsames Merkmal das die Unternehmen auszeichnet ist die starke Prägung durch den Unternehmer. Es handelt sich um KMU die wettbewerbsfähig auf ihrem Markt und auch genügend innovativ sind. Diese KMU konzentrieren sich stark auf die Differenzierungsstrategie und auf die Dienstleistungen. Sie sind gewohnt in der Planung der Wachstumsphasen, Strategien zu formulieren, für mittel- und langfristige Zeitperioden.

\begin{abstract}
Managers of successful organisations in both the private and public sectors of advanced countries share a dirty little secretthey don't understand why they are successful. Our technologies, economy, and society are so complex that it is impossible for single individuals to fully understand them.
\end{abstract}

(RYCROFT et Kash, 1999, p. 3).

\title{
Introduction
}

Depuis les années 1980, on observe que les chercheurs et les décideurs en Europe ont tous recommencé à s'intéresser fortement aux thèmes de la création, de la croissance et de la compétitivité des PME (DTI, 1996; OCDE, 1997, 2000 ; Odaka et Sawai, 1999). La mondialisation des marchés, la dématérialisation des productions et la flexibilité des processus sont des tendances fortes d'une économie post-fordiste qui est en train d'évoluer rapidement et qui n'est pas encore déterminée (Taranto, 1990 ; Veltz, 1996 ; Rullani et Romano, 1998).

Cet intérêt, particulièrement marqué en Italie (Golfetto, 1988 ; Mussati, 1990 ; AA.VV, 1995), relève de deux considérations qui dérivent de données réelles :

1. L'importance des PME en Italie est décisive ; cette importance est certainement plus grande que dans beaucoup d'autres pays industrialisés (voir OCDE, 2000) : les PME représentent plus du $95 \%$ des entreprises, $60 \%$ des employés, $45 \%$ du chiffre d'affaires et $34 \%$ de l'exportation nationale.

2. Dans la dernière décennie, les PME se sont montrées les premières créatrices d'emplois. Tout en ayant besoin de soutiens extérieurs pour se renouveler et être concurrentielles, elles ont eu des succès étonnants. Cela ne veut toutefois pas dire qu'il n'y ait pas de nuages dans cet horizon de l'économie. Il faudra donc intervenir avec des politiques appropriées afin que leurs lacunes structurales ne deviennent pas des obstacles futurs à la croissance.

Revue internationale P.M.E., vol. 14, $\mathrm{n}^{\text {os }} 3-4,2001$ 
Le présent travail est le résultat d'un plus vaste projet de recherche internationale (Storey, 1997; MEA, 1999b; OCDE, 2000) mené en collaboration avec l'OCDE ${ }^{2}$ - il précise le rôle des PME dans la création de nouveaux postes de travail en Italie (Pencarelli, 1995; Mutinelli, 1997; Corbetta, 2000). En même temps, il reconstruit, en les interprétant, les parcours qui ont été suivis par le sous-ensemble de PME qui a crû le plus dans la première moitié des années 1990.

La structure de ce travail est la suivante. La première partie expose la méthodologie utilisée. On présente brièvement les choix les plus importants, tout en rappelant comment le groupe de coordination de $\mathrm{l}^{\prime} \mathrm{OCDE}^{3}$ en a discuté et comment il les a approuvées.

Une définition fondamentale est celle d' «entreprise pérenne », c'est-à-dire une entreprise dont la présence dans le marché est d'au moins six ans. On résume, dans la deuxième partie, la contribution de ces entreprises à la création d'emplois, en les divisant selon leur taille, leur secteur d'activité et leur localisation sur le territoire. En utilisant un indice de croissance convenu, l'indice Birch, que l'on applique à la donnée sur les employés, on distingue les PME en croissance de celles à forte croissance et on décrit leur articulation telle que l'on en a précédemment discuté (section 2.1).

La troisième partie porte sur une analyse qualitative menée sur un vaste échantillon d'entreprises pérennes en croissance. L'analyse identifie quatre types d'entreprises en croissance - les jeunes, les exportatrices, les dominées et celles qui appartiennent à un groupe - qui ont des comportements relativement homogènes par rapport aux performances de croissance obtenues (section 4).

À partir de la théorie des processus de croissance, la cinquième partie permet d'identifier trois séries de facteurs - externes, internes et les motivations personnelles du dirigeant -, qui sont fréquemment évoqués pour expliquer la croissance des PME, et ainsi de vérifier comment ils se manifestent dans les quatre groupes d'entreprise précédemment identifiés.

Certains résultats de l'analyse qualitative nous amènent à conclure en évoquant les politiques qui seraient les plus appropriées pour soutenir la croissance, tout en faisant état des difficultés qui peuvent se poser.

2. L'équipe italienne qui a analysé le cas italien a travaillé au sein du CERTeT (Centre d'économie régional, des transports et du tourisme) de l'Université Bocconi de Milan, sous la direction d'Alberto Bramanti.

3. Pour approfondir la méthodologie d'analyse utilisée, le lecteur pourra consulter les documents de travail et de synthèse du projet.

Revue internationale P.M.E., vol. 14, $\mathrm{n}^{\text {os }} 3-4,2001$ 


\section{La méthodologie}

L'univers des entreprises italiennes avec au moins un employé en 1995 comprend 911366 entreprises, procurant du travail à 10032555 employés, soit une moyenne de 11 employés par entreprise. De ce stock d'entreprises, on a extrait le sousensemble de celles qui étaient actives en 1990; il s'agit donc d'entreprises âgées d'au moins six ans et que, dans ce travail-ci, on appelle les «entreprises pérennes». En ce qui concerne l'Italie, on parle de 552795 entreprises au total avec 7772257 employés et donc, légèrement plus grandes, avec 14 employés chacune. Si l'on applique le filtre de la taille (de 20 à 499 employés $^{4}$ ), on se restreint à 45171 entreprises ayant 2659208 employés, soit 59 employés chacune.

Les taux de survie de ces entreprises - réparties par classe de taille et par ancienneté - confirment des travaux précédents (Mussati, 1990; Biggiero et Caroli, 1995). Les entreprises ayant au moins six ans représentaient, en effet, $87,7 \%$ du stock des PME qui existaient en 1995. Si l'on considère les entreprises d'au moins dix ans d'ancienneté, on atteint $76 \%$ de ce stock.

L'indice de Birch (1987) mérite une explication, quoique brève. Cet indice est l'algorithme servant à calculer les taux de croissance des entreprises pendant les six ans considérés (1990-1995); il permet de calculer les valeurs limites distinguant les entreprises à forte croissance de celles simplement en croissance. On définit cet indice de la façon suivante :

$$
\text { Birch }=\left(\mathrm{emp}_{95}-\mathrm{emp}_{90}\right) \cdot \mid\left(\mathrm{emp}_{95}-\mathrm{emp}_{90}\right) / \mathrm{emp}_{90} \mathrm{l}
$$

La présence de la valeur absolue permet à l'indice d'assumer aussi des valeurs négatives - lorsque l'entreprise effectue des licenciements - tandis que le produit de la variation absolue par la variation relative, balance les effets que la taille de l'entreprise a sur les taux de croissance - les grandes entreprises en croissance ont tendance à enregistrer des variations absolues significatives, tandis que les petites entreprises en croissance mettent en évidence des variations relatives significatives ${ }^{5}$.

4. Pour permettre une comparaison sur le plan national, celle-ci est la définition de PME que l'on a retenue dans l'étude de l'OCDE. Pour le cas italien, on a aussi analysé les entreprises de 10 à 19 employés.

5. L'indice n'est pas sans défauts et il se prête à des modifications éventuelles ad hoc pour considérer la structure industrielle spécifique que l'on analyse. Après l'avoir calculé pour les entreprises permanentes - d'après ce que l'on a établi lors de l'étude de l'OCDE -, toutes les entreprises en croissance du secteur manufacturier ont été divisées par les volumes décroissants de l'indice Birch. On a choisi de considérer le niveau de $5 \%$ comme étant un taux élevé de croissance (les gazelles); la valeur numérique qui détermine le premier $5 \%$ des autres entreprises permanentes en croissance vaut, au cas italien, 150 ; c'est le seuil qui distingue les PME à forte croissance de toutes les autres.

Revue internationale P.M.E., vol. 14, $\mathrm{n}^{\text {os }} 3-4,2001$ 
Dans la deuxième phase de la recherche, on a retenu un échantillon ${ }^{6}$ de PME en croissance (et autant que possible en forte croissance) pour les soumettre à une analyse qualitative, afin de connaître leurs spécificités, leurs modèles d'organisation et leurs stratégies. Elle repose sur un questionnaire structuré ${ }^{7}$ et divisé en cinq sections portant sur les thèmes suivants :

a) les caractéristiques de l'entreprise et le scénario compétitif;

b) les profils de croissance et la gestion des ressources humaines;

c) les modèles d'organisation et leur orientation vers la croissance;

d) les relations avec l'extérieur et les partenariats ;

e) le rôle des institutions et les politiques d'encouragement.

Il a été possible de mettre en évidence les caractéristiques des trajectoires de croissance, les sentiers de développement suivis par chaque entreprise et, enfin, de vérifier certains groupes de facteurs que la théorie (Karlsson, Johannison et Storey, 1993) identifie comme les seuls capables d'expliquer la croissance:

a) les facteurs internes - le modèle d'organisation, la gestion des ressources humaines et des décisions stratégiques (Mussati, 1990; Harhoff, Stahl et Woywode, 1996; Arcari, 1999);

b) les facteurs externes et ceux liés au milieu - les tendances du marché, les relations avec les partenaires et avec les institutions (Audretsch, 1995; Curran, Stanworth et Watkins, 1986 a et b ; Johnson, Baldwin et Hinchley, 1997);

c) le rôle de l'entrepreneur sur la croissance (Brock et Evans, 1986; Lewis, Stanworth et Gibb, 1984 ; Marchini, 1995a ; MEA, 1999a).

Les résultats ont été traités avec le logiciel de statistique SPSS ${ }^{\mathrm{TM}}$, qui nous a permis de faire des analyses descriptive, typologique et multivariée. Comme les tests statistiques se sont souvent révélés insatisfaisants, nous avons décidé d'en

6. L'échantillon italien est constitué de 414 entreprises, situées dans sept différentes régions : le Piémont, la Lombardie et l’Émilie-Romagne au nord; les Marches au centre ; les Pouilles, la Basilicate et la Sardaigne au sud.

7. Le questionnaire a été soumis par des chercheurs expérimentés aux entrepreneurs ou aux chefs d'entreprise qui ont été interviewés au cours d'une entrevue d'une heure et demie environ. En ce qui concerne les politiques, on a interviewé différents interlocuteurs privilégiés, qui ont discuté de leur vision des PME et commenté les résultats de l'étude avec les entrepreneurs (on a eu entre 5 et 8 contacts dans chaque région où l'on a mené l'étude, soit un total de 40 contacts). 
présenter le contenu selon une approche qualitative ${ }^{8}$, qui tend à proposer et à généraliser des résultats ayant été confirmés également par des décideurs et des interlocuteurs privilégiés.

\section{La création d'emplois}

Dans la première moitié des années 1990, les PME pérennes italiennes (20-499 employés) ont accru leur effectif pour un solde net de 55408 employés. Ce solde s'élève considérablement si on élargit le champ d'observation pour tenir compte du sous-ensemble des entreprises ayant de 10 à 19 employés. La création nette d'emplois de cette classe est, en effet, de 34732 nouveaux postes, pour un total de 90140 emplois pour les entreprises ayant entre 10 et 499 employés.

Pour les PME pérennes, les employés ont augmenté en moyenne de $4 \%$. Dans le cas des secteurs manufacturiers et des services, cette croissance était respectivement de $3,2 \%$ et de $11 \%$. Ce taux moyen se ressent fortement du déclin de la classe des entreprises moyennes (200-499) qui, dans la période 1990-1995, a perdu presque 14000 emplois. Il faut aussi signaler la performance particulièrement forte de la classe des 50-99 employés qui affiche les taux de croissance les plus élevés, tant dans le secteur manufacturier que dans celui des services.

L'analyse des entreprises pérennes par territoire révèle l'importance pour la création d'emplois de toutes les macrorégions ${ }^{9}$, sauf le Sud et surtout, le Sud-Ouest, où même les plus petites entreprises (10-19 employés) ont créé peu d'emplois contrairement aux autres régions italiennes.

Tant pour le manufacturier que pour les services aux entreprises, la région qui a créé le plus d'emplois est le Nord-Est (respectivement $+5,6 \%$ et 19,1\%); seules les entreprises moyennes ont perdu des emplois dans toutes les zones, y compris le Nord-Est, à l'exception du Nord-Ouest (Banco Ambrosiano Veneto, 1996).

Les autres régions se différencient du point de vue manufacturier et services aux entreprises. Le manufacturier augmente en effet significativement au NordOuest (+ $3 \%)$ et de façon modérée au Centre, tandis qu'il décroît fortement au Sud

8. Pour une présentation de quelques résultats de régression et d'analyse cluster, le lecteur consultera les rapports de synthèse de l'équipe du CERTeT.

9. Le territoire italien entier est découpé en cinq macrorégions : le Nord-Ouest, composé par le val d'Aoste, le Piémont, la Ligurie et la Lombardie; le Nord-Est, la Vénétie, le Trentin-Haut-Adige, le Frioul-Vénétie Julienne et l'Émilie-Romagne ; le Centre, composé par la Toscane, les Marches, l'Ombrie et le Latium ; le Sud-Est, les Abruzzes, la Molise, les Pouilles et la Basilicate; et le Sud-Ouest, composé par la Campanie, la Calabre, la Sicile et la Sardaigne.

Revue internationale P.M.E., vol. 14, $\mathrm{n}^{\text {os }} 3$-4, 2001 


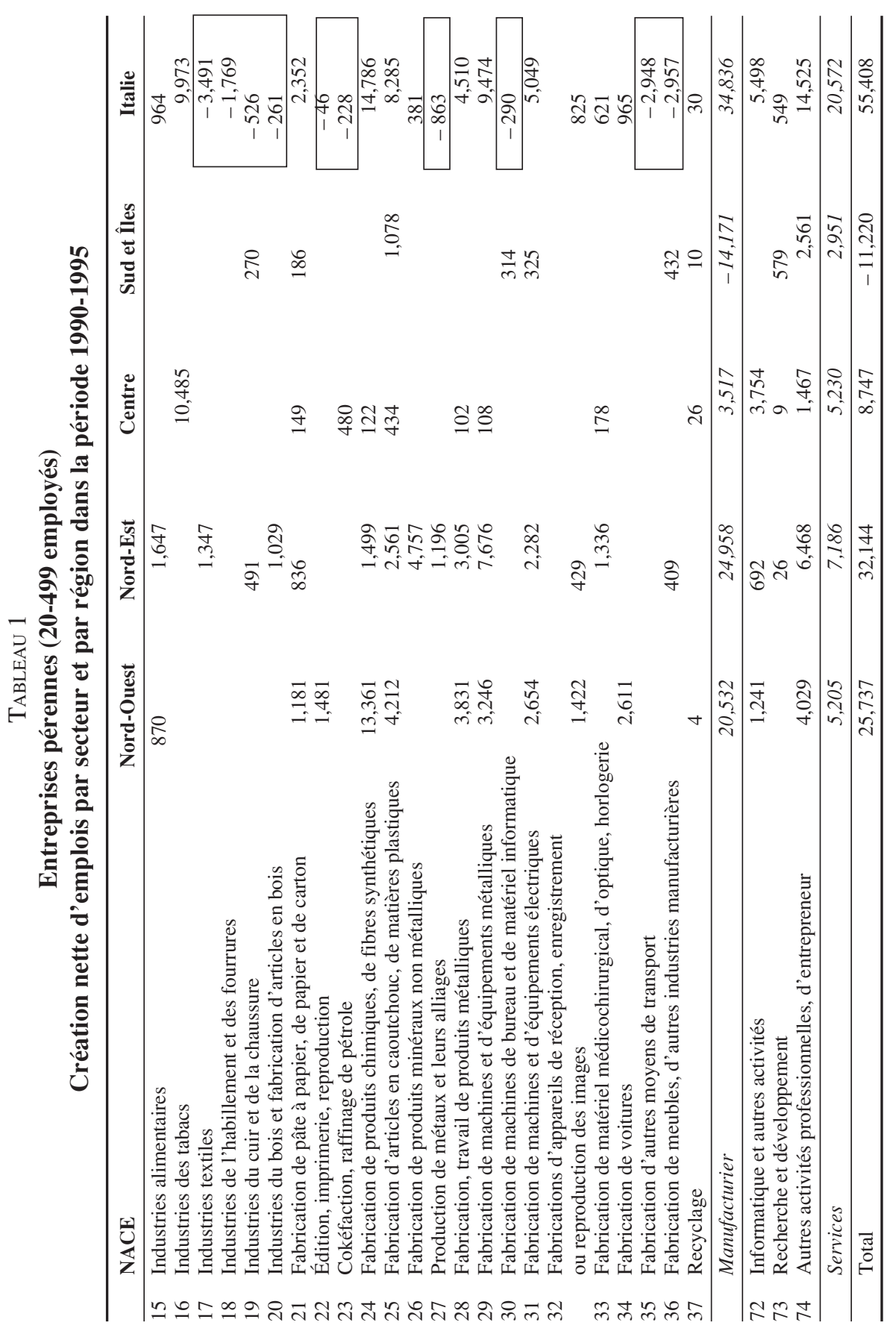

Revue internationale P.M.E., vol. 14, $\mathrm{n}^{\text {os }} 3-4,2001$ 
(au total-8,6\%). Les services augmentent partout, mais de façon particulièrement forte au Centre $(+11,8 \%)$, au Sud $(7 \%)$ et, de façon moindre, au Nord-Ouest $(+6,7 \%)$.

L'analyse, par secteur d'activité des petites et moyennes entreprises pérennes permet de brosser un tableau beaucoup plus varié (voir tableau 1). Dans quelques secteurs industriels, les nouveaux postes de travail créés par les entreprises en croissance n'ont pas suffi à racheter la perte. Cela est vrai même là où la contribution des PME à forte croissance a été plus élevée qu'ailleurs.

Les cinq principaux secteurs ${ }^{10}$, selon la valeur absolue des emplois créés au cours de la période, sont les suivants : 24 (Fabrication de produits chimiques, de fibres synthétiques), 25 (Fabrication d'articles en caoutchouc, de matières plastiques), 28 (Fabrication, travail de produits métalliques), 29 (Fabrication de machines et d'équipements métalliques) et 31 (Fabrication de machines et d'équipements électriques). Ensemble, ils ont créé plus de 42000 emplois, soit $76 \%$ du total manufacturier. Ces industries aux biens intermédiaires et aux biens instrumentaux qui sont à la tête des productions légères du Made in Italy ont aussi réalisé des performances à l'exportation fort intéressantes dans les années 1990 (Quadrio Curzio et Fortis, 2000).

Au cours de la même période, on enregistre un processus de diffusion territoriale des secteurs dont on a parlé. Il s'agit d'un phénomène que l'on analyse en considérant la variation de l'indice de concentration de Herfindal, qui décroît au cours de la période quinquennale, par rapport aux employés, dans tous les secteurs et, par rapport aux entreprises, selon quatre des cinq secteurs. On en arrive à une forte concentration de secteur (comme dans d'autres contextes nationaux, notamment aux États-Unis, en Allemagne et en Suède), coïncidant avec une concentration territoriale aussi forte : 27304 postes de travail - qui équivaut au $65 \%$ du total sectoriel - ont été créés au Nord-Ouest.

\subsection{Les PME en croissance}

Si l'on analyse le sous-ensemble des entreprises en croissance, on peut comprendre leur contribution à la création d'emplois. Les quelque 29000 entreprises pérennes (20-499) se repartissent entre 10500 entreprises en croissance, 6100 stables et près de 12500 en contraction. Ces 10500 entreprises en croissance sont, toutefois, responsables de la création de 321500 nouveaux emplois. Ce chiffre monte à plus de 425000 lorsque l'on considère les entreprises plus petites (10-19). Le tableau 2 montre cet effet net.

10. Le secteur 16 (Industries des tabacs) représente une anomalie statistique, étant donné qu'il y a eu une concentration d'employés dans l'établissement des sociétés d'État.

Revue internationale P.M.E., vol. 14, $\mathrm{n}^{\text {os }} 3-4,2001$ 


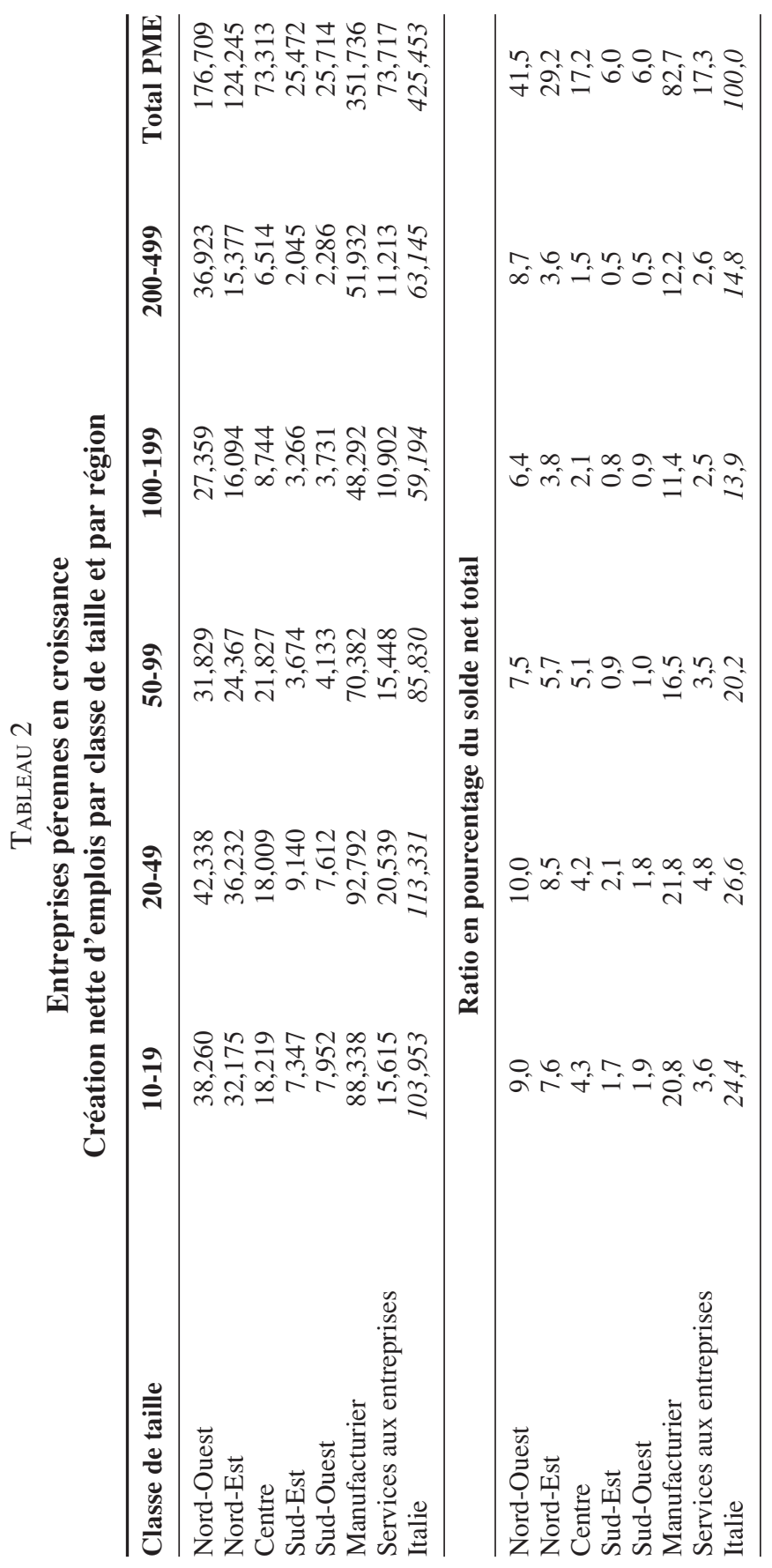

Revue internationale P.M.E., vol. 14, $\mathrm{n}^{\text {os }} 3-4,2001$ 
En parcourant les colonnes, on voit que les deux premières classes, considérées ensemble, créent plus de $50 \%$ du total des nouveaux emplois, dont $42,6 \%$ par le manufacturier. Les entreprises de la classe 20-49 ont créé au cours de la période considérée plus de 113000 emplois, donc beaucoup plus que toutes les entreprises pérennes.

La lecture par lignes révèle l'excellente performance du secteur manufacturier - presque 352000 nouveaux emplois - réalisée au cours d'une période de temps où généralement l'industrie perdait des emplois (Istat, 2000). Le Nord-Ouest est la région dont la croissance des entreprises a été la plus forte, et de loin, et qui enregistre un solde net total supérieur à n'importe quelle autre zone $(41,5 \%)$.

L'emploi dans les entreprises en croissance est mieux réparti dans toutes les classes de taille, même si le rôle des plus petites entreprises est toujours très important (AA.VV., 1995 ; Solinas, 1996) - cette situation est la même dans toutes les zones du territoire; mais au Nord, le comportement des entreprises moyennes est encore meilleur, en raison notamment de ces dernières (presque 37000 nouveaux emplois).

\subsection{Les $\mathrm{PME}$ à forte croissance}

Les PME à fort taux de croissance, soit $5 \%$ des entreprises pérennes en croissance, tout en étant peu nombreuses, offrent une contribution substantielle à la création d'emplois. Parmi les 10500 entreprises en croissance (20-499) au cours de la période, 409 peuvent être considérées comme des «gazelles », créant 125274 emplois nets additionnels, soit 33,5\% du total des emplois créés par les entreprises en croissance.

Le tableau 3 permet d'apprécier leur performance. En jetant un coup d'œil sur les colonnes, où les entreprises sont classées selon leur taille, on voit que la part d'emplois créés est plus grande chez les entreprises moyennes, 200-499 employés $(26,3 \%)$, suivie de la classe 50-99 employés (26,2\%). La contribution des plus petites entreprises (10-19 employés) est plus faible, soit $12 \%$ du total des nouveaux emplois. Ce résultat est encore plus important si l'on compare le pourcentage des nouveaux emplois dans les entreprises à forte croissance à celui des nouveaux emplois créés dans les entreprises en croissance.

La lecture par lignes du tableau 3 souligne, encore une fois, le rôle prédominant du Nord-Ouest - dont la contribution à la création d'emplois est presque de $45 \%$ - et, par contraste, le faible rôle du Nord-Est. Les dimensions des deux zones géographiques de même que les performances variables des entreprises à forte croissance peuvent expliquer ce résultat; on notera également que les pourcentages pour le Nord-Est sont inférieurs pour chaque classe de taille.

Revue internationale P.M.E., vol. 14, nºs 3-4, 2001 


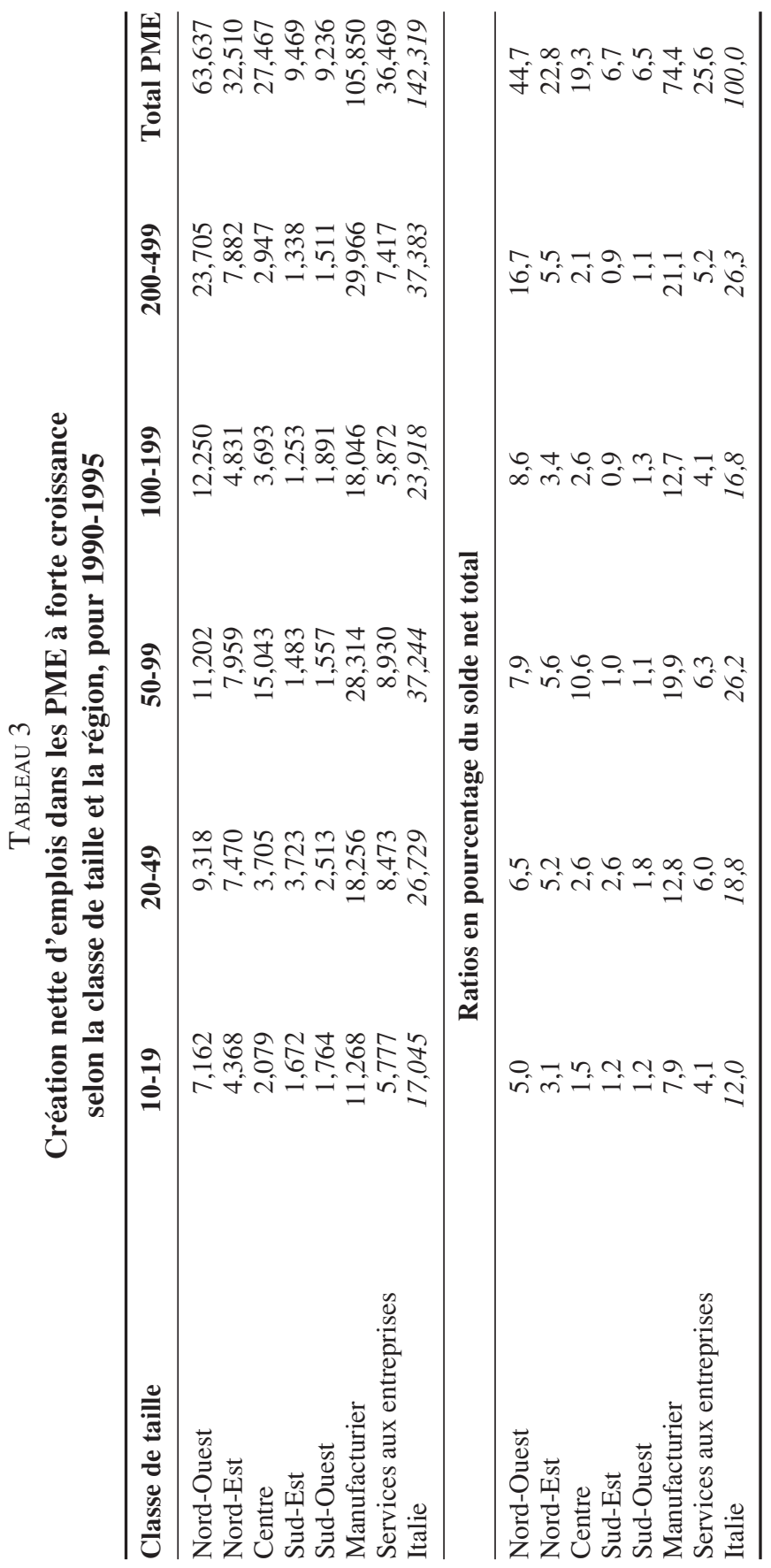

Revue internationale P.M.E., vol. 14, $\mathrm{n}^{\text {os }} 3-4,2001$ 
L'importance du Centre et du Sud (du Sud-Est plus que du Sud-Ouest) est aussi significative : en retenant les valeurs absolues modérées, les entreprises à forte croissance apportent une contribution notable à la création d'emplois.

Sur le plan des secteurs, la contribution à l'augmentation des emplois par les PME à forte croissance a été plus grande dans les services (49,5\%) que dans l'industrie $(30,1 \%)$. Les ratios sont systématiquement plus élevés pour chaque classe de taille. En outre, la contribution du secteur des services met en évidence une distribution géographique beaucoup plus équilibrée, plus forte dans le NordOuest $(31 \%)$ et plus également répartie dans les trois autres zones géographiques, coïncidant avec les secteurs dont l'impact net d'occupation est le plus fort, sauf le secteur 22.

Dans l'industrie manufacturière, il faut signaler les secteurs suivants : 24, Fabrication de produits chimiques, de fibres synthétiques (66,3\% de l'augmentation nette des emplois dans les entreprises pérennes provient des PME à forte croissance); 22, Édition, imprimerie, reproduction (51,9\%); 31, Fabrication de machines et d'équipements électriques (50,2\%).

\subsection{Vers une première synthèse}

La faible croissance de l'économie italienne dans la première moitié des années 1990 n'a évidemment pas eu un impact positif sur l'emploi (ISTAT, 2000). Le secteur manufacturier $a$, en effet, perdu près de 121000 emplois. En comparaison, on relève la création de 90000 emplois par les entreprises pérennes (10-499 employés). Ce solde augmente encore et de façon notable, si l'on étend l'analyse à toutes les tailles d'entreprises.

Mais on note des points négatifs dans les entreprises pérennes : dans certains secteurs, principalement dans les secteurs à forte intensité de main-d'œuvre et du Made in Italy, dans certaines régions (surtout au Sud) et certaines classes de taille (surtout les 200 à 499 employés), on a eu tendance à effectuer des licenciements.

La reprise conjoncturelle de 1993 - due à l'augmentation de l'exportation, conséquemment à la dévaluation de la lire en 1992 - a obligé les PME à être plus flexibles, particulièrement celles situées au Nord et celles œuvrant dans des secteurs qui reposent sur la production des biens intermédiaires et des biens capitaux (Anastasia et Corò, 1996; Banco Ambrosiano Veneto, 1996).

À l'échelon territorial, le ratio d'entreprises pérennes de loin le plus important se situe au Nord, tandis qu'au Centre et au Sud la présence de PME à forte croissance (les gazelles) est significative par rapport à celle des PME pérennes.

Revue internationale P.M.E., vol. 14, $\mathrm{n}^{\text {os }} 3-4,2001$ 


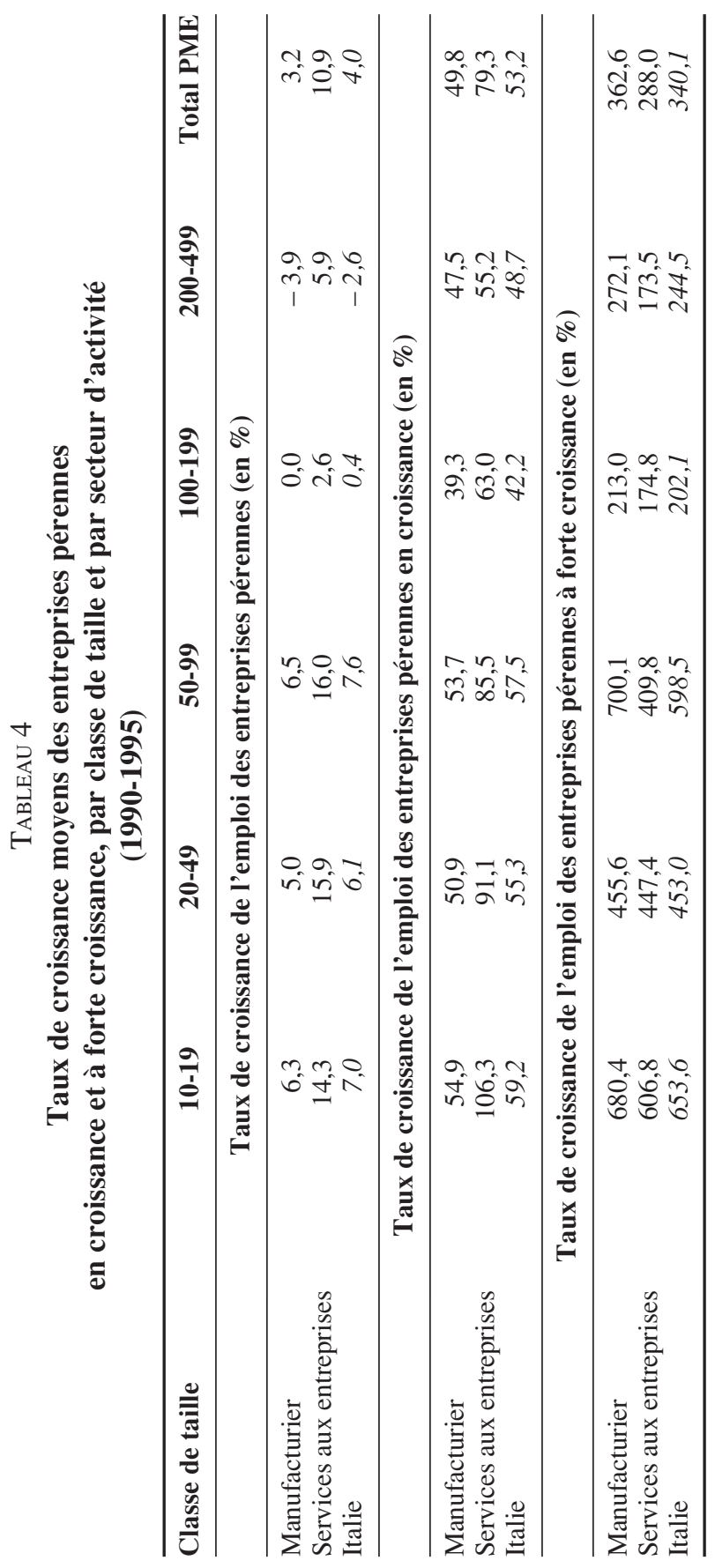

Revue internationale P.M.E., vol. 14, $\mathrm{n}^{\text {os }} 3-4,2001$ 
À ce point de la réflexion, on peut déjà proposer deux applications importantes pour les politiques. Les trajectoires de croissance sont, avant tout, multiformes et bigarrées ; il n'y a, en outre, aucune invariance statistique forte. On ne peut donc pas conclure que l'entreprise en croissance se concentre dans certaines classes dimensionnelles, dans certains territoires ou certains secteurs ${ }^{11}$. Ensuite, le problème des régions les plus faibles est évidemment d'aider les entreprises à survivre (puisque la création d'entreprises est forte partout en Italie) : les entreprises pérennes pourront ainsi croître un peu partout, y compris au Sud, comme on l'a vu. Le défi des politiques est de passer de l'aide à la création à l'aide à la pérennisation, pour accompagner les PME tout au long de leur croissance.

\section{L'analyse qualitative des PME à forte croissance}

Après avoir présenté les données statistiques se rapportant aux entreprises pérennes et en croissance, dans la seconde partie de cette analyse, on se concentre sur les entreprises en croissance, pour faire une analyse qualitative des raisons de cette croissance. Rappelons que l'échantillon se compose de 414 PME pérennes en croissance, dont 117 à forte croissance et 257 en croissance, réparties dans six régions italiennes.

La plupart de ces entreprises à forte croissance ou en croissance ont été créées après 1980. Dans le secteur manufacturier, les entreprises à forte croissance sont normalement plus jeunes, puisque $34 \%$ d'entre elles ont été lancées en 1990. Le fort taux de croissance de ces « jeunes » entreprises peut être dû à la présence d'une occasion à saisir lors du lancement en affaires et à la nécessité d'atteindre rapidement une bonne taille (Biggiero et Caroli, 1995; Arcari, 1999). Étant donné que l'échantillon est composé d'entreprises qui ont de toute façon cinq ans, le sousgroupe créé en 1990 est justement formé des entreprises qui ont réussi à se maintenir sur le marché, tout en surmontant les difficultés courantes des premières années d'activité, là où la mortalité est souvent plus élevée.

Dans le domaine des services aux entreprises, les différences entre les entreprises à forte croissance et celles en croissance sont moins nettes; cependant, les premières sont un peu plus jeunes ( $87 \%$ des entreprises à forte croissance sont nées après 1980 , contre $80 \%$ pour les entreprises en croissance). En ce qui concerne la distribution de ce secteur dans l'échantillon, on peut voir tous les liens avec le secteur manufacturier. Ces services comprennent aussi le transport, la consultation et l'aide technique et les autres activités.

L'analyse de l'échantillon permet de relever quelques différences entre le secteur manufacturier et celui des services aux entreprises, de même qu'entre les

11. La conséquence de cette constatation est l'insatisfaction que procure une analyse multivariée de l'échantillon.

Revue internationale P.M.E., vol. 14, nºs 3-4, 2001

(C) 2002 - Presses de l'Université du Québec

Édifice Le Delta I, 2875, boul. Laurier, bureau 450, Sainte-Foy, Québec G1V 2M2 • Tél. : (418) 657-4399 - www.puq.uquebec.ca

Tiré de : Revue internationale P.M.E., vol. 14, $\mathrm{n}^{\text {os }} 3-4$, sous la direction de Pierre-André Julien. 
entreprises à forte croissance et en croissance. Ces différences se rapportent à la taille (employés et chiffre d'affaires), à l'exportation, à l'appartenance à un groupe et à la dépendance et enfin, à une clientèle peu nombreuse.

La taille moyenne des entreprises à forte croissance est généralement inférieure à celle des entreprises en croissance en 1990; mais, grâce à leur croissance plus rapide, elles les dépassent à la fin de la période. Cette caractéristique met en évidence des parcours de croissance plus dynamiques pour ces entreprises qui doivent atteindre des tailles opérationnelles plus fortes pour maintenir leur compétitivité (Karlsson, Johannisson et Storey, 1993; Solinas, 1996).

Cette forte augmentation des emplois dans les entreprises manufacturières à forte croissance entraîne pourtant une réduction de la productivité (chiffre d'affaires / employés) par opposition à de faibles augmentations de la productivité pour les entreprises en croissance (Centrale dei Bilanci, 2000). Cette tendance est différente pour les services aux entreprises, où une augmentation du nombre d'employés n'implique pas une réduction de la productivité. Cette donnée confirme l'existence de différences dans les processus de croissance dans ces deux grands secteurs, avec une augmentation des coûts fixes plus élevée dans le secteur manufacturier ${ }^{12}$.

Parmi les entreprises analysées, le groupe de celles qui exportent est assez peu nombreux : ce groupe comprend près de $26 \%$ des entreprises concentrées principalement dans le secteur manufacturier. Comme la documentation l'a déjà mis en évidence (Storey, 1995), le lien entre l'exportation et la croissance d'entreprise n'est pas toujours fort. L'exportation moyenne des entreprises manufacturières à forte croissance augmente, cependant, de plus de cinq fois entre 1990 et 1996, alors que pour les entreprises en croissance, cette augmentation est plus faible. En même temps, la croissance du ratio moyen d'exportation par rapport au chiffre d'affaires est semblable dans les deux groupes ; cela signifie que même le chiffre d'affaires des entreprises à forte croissance augmente très rapidement.

Un autre élément qui caractérise les entreprises est leur dépendance plus ou moins grande envers leurs clients. Cela est mesuré par la part du chiffre d'affaires réalisé avec les trois premiers clients : lorsque cette part est élevée, l'entreprise coeteris paribus - a un pouvoir contractuel réduit et donc, s'expose à plus de risques. Dans cette situation, la croissance de l'entreprise peut être liée à des variations de court terme, dues aux commandes de peu de clients (les pics de la demande). La situation d'une entreprise qui peut répartir le risque entre plusieurs clients est différente: sa croissance se caractérise par une plus grande stabilité et peut être considérée comme le résultat d'une stratégie d'expansion différente sur le marché.

12. Ces considérations confirment que la croissance avance par étapes : une saturation de la capacité de production, de nouveaux investissements, des engagements, puis, la consolidation. Généralement, la rentabilité diminue de plus en plus tout de suite après le saut de taille, en raison des nouveaux coûts fixes, pour remonter par la suite (Dellacasa, 1996).

Revue internationale P.M.E., vol. 14, $\mathrm{n}^{\text {os }} 3-4,2001$ 
Un dernier élément important qui a été considéré dans le questionnaire est l'appartenance éventuelle de l'entreprise à un groupe et le rôle de celui-ci. En 1990, la part des entreprises à forte croissance qui appartiennent à un groupe est généralement plus grande par rapport à celle que l'on observe chez les entreprises en croissance. Cette part, qui est la même pour le manufacturier et les services aux entreprises, augmente entre 1990 et 1996 ; cela indique un processus d'acquisition des «gazelles » par des groupes existants ou, ce qui est encore plus significatif, la création de groupes par les mêmes entreprises à forte croissance.

La position hiérarchique de l'entreprise à l'intérieur du groupe révèle son importance : $46 \%$ des entreprises manufacturières à forte croissance et $69 \%$ des entreprises de services à forte croissance ont un rôle de «contrôle »; ces pourcentages sont, en comparaison, respectivement $27 \%$ et $33 \%$ pour les entreprises en croissance.

En résumé, cette analyse préliminaire permet d'affirmer que, en moyenne, les entreprises à forte croissance :

a) sont plus jeunes que celles en croissance;

b) s'orientent beaucoup plus vers l'exportation et l'augmentation de leurs parts d'exportation (avec des politiques de pénétration de marchés, composé aux entreprises en croissance);

c) ont un chiffre d'affaires soutenu par plus de clients par rapport aux entreprises en croissance;

d) appartiennent plus fréquemment à un groupe et détiennent une position de contrôle dans celui-ci.

\section{Quatre groupes de PME à forte croissance}

Après diverses analyses, telles des analyses de régression pour expliquer le taux de croissance des entreprises, à partir des variables quantitatives disponibles, sans obtenir des résultats positifs du point de vue statistique, on a poursuivi la recherche sur la structure et les comportements, ce qui nous a permis de diviser l'échantillon selon les comportements similaires en ce qui concerne les trajectoires de croissance.

Pour limiter et mieux contrôler la variété d'ensemble, on se concentre ici sur le sous-ensemble manufacturier (les différences qu'elles ont avec les entreprises des services sont, en effet, évidentes) ; cela nous permet de diviser les $75^{13}$ cas en quatre sous-groupes :

13. L'échantillon total est le suivant : 117 entreprises à forte croissance (75 manufacturières et 42 dans le secteur des services aux entreprises), 297 entreprises en croissance (230 manufacturières et 67 dans les services).

Revue internationale P.M.E., vol. 14, nºs 3-4, 2001 
a) les entreprises « jeunes » (18 entreprises), pour qui la croissance est indispensable pour atteindre une taille optimale; elles ont fréquemment à leur tête des entrepreneurs plus jeunes que la moyenne et qui se distinguent par une forte propension à l'innovation (Johnson, Baldwin et Hinchley, 1997; MEA, 1998);

b) les «exportatrices» (18 entreprises), qui sont beaucoup plus solides, occupent une bonne position sur le marché et sont capables de profiter d'une poussée de la demande avec l'étranger chaque fois qu'elles en ont l'occasion, mais elles sont aussi capables de consolider avec le temps leurs propres positions sur le marché, tout en poursuivant de véritables parcours d'internationalisation (EFER, 1996; Galli, 1999);

c) les « dominées » (25 entreprises), où la croissance se maintient, au contraire, grâce aux commandes d'un petit nombre de clients et, pour cela, elles peuvent sembler plus instables;

d) les « appartenantes à un groupe » (14 entreprises), où l'exploitation d'économies de groupe externes, surtout celles qui sont en relation avec le secteur financier et avec les maisons de commerce, est importante (Mutinelli, 1997).

Ces quatre groupes ont certaines caractéristiques qui peuvent se retrouver en partie chez les autres, mais elles ont une caractéristique dominante ${ }^{14}$. Ainsi, les entreprises «jeunes» et celles «dominées » ont une structure plus faible par rapport aux deux autres catégories. Les « jeunes » sont en train de traverser la phase extrêmement délicate de la consolidation du marché et cherchent à atteindre une dimension optimale; les « dominées » ont lié leur propre évolution à celles de leurs clients les plus importants et doivent donc veiller à se diversifier.

Les différenciations parmi les groupes sont quelquefois significatives, mais leur trajectoire de croissance comprend aussi des éléments communs, soit l'importance accordée à la qualité des produits et des services offerts, aux ressources humaines (il y a un fort intérêt envers l'engagement de jeunes pour qu'ils acquièrent une première expérience de travail) et, enfin, à l'apport des fournisseurs.

14. L'attribution de cette « dominance » n'est pas possible dans tous les cas, aussi quelques entreprises ont donc été exclues et l'on a travaillé au total avec environ 70 cas. Le nombre de cas ainsi traités ne permet pas d'articuler la lecture des caractéristiques des entreprises et des comportements ni par secteur, ni par microrégions. 


\section{Les explications de la croissance : les facteurs externes, les facteurs internes et les motivations d'entrepreneurs}

Après avoir identifié ces quatre familles d'entreprises, on analysera le comportement des entreprises mêmes, en le confrontant aux facteurs précisés par la théorie expliquant la croissance (Hull et Hjern, 1987 ; Karlsson, Johannisson et Storey, 1993 ; Storey, 1995 ; DTI, 1996; Arcari, 1999). On peut diviser ces facteurs en trois groupes, soit les facteurs externes (section 5.1), les facteurs internes (section 5.2) et le comportement de l'entrepreneur à l'égard de la croissance (section 5.3). On en discutera rapidement, tout en ayant conscience des limites de chacun et du fait que les processus sont toujours circulaires et relèvent de nombreuses interactions, de conditionnements réciproques et de retours en arrière. Le croisement de ces facteurs de croissance et des groupes d'entreprises complétera le tableau de l'analyse et permettra de relever les principaux points communs et différences entre les groupes.

\subsection{Les facteurs externes}

Un premier groupe de facteurs comprend ceux qui définissent l'arène de compétition dans laquelle l'entreprise agit et qui ne peuvent être influencés que de façon marginale par les décisions internes (Taranto, 1990; AA.VV., 1995 ; Audretsch, 1995 ; Johnson, Baldwin et Hinchley, 1997 ; Corbetta, 2000). On trouve ici, particulièrement, les évaluations des entreprises par les cours du marché et leurs positions concurrentielles (sous-section 5.1.1), par leurs relations avec les partenaires et avec d'autres entreprises (sous-section 5.1.2) et par le rapport des offices et institutions locales (sous-section 5.1.3).

\subsubsection{Positions concurrentielles}

Dans ce premier groupe de mesures, on trouve évidemment plusieurs convergences d'évaluation. Avant tout, l'orientation vers le marché intérieur est forte, avec un chiffre d'affaires moyen réalisé sur le marché italien supérieur à $80 \%$, sauf évidemment pour le groupe des entreprises qui exportent au moins $50 \%$ de leur production. Le marché national est donc important dans les trajectoires de croissance des PME, car il permet une mise au point des produits plus rapide, le choix de quelques clients semblables du point de vue culturel et géographique, une interaction avec les producteurs en fournissant ces précieux feed-back qui dérivent des processus d'apprentissage par l'expérience. Ce marché permet aussi d'économiser les précieuses ressources de l'entreprise que l'on devrait allouer à la gestion de marchés lointains, souvent plus difficiles. À ce sujet, on relève, du reste, un considérable défi stratégique, car il est évident que déjà le concept de «marché national » s'élargit au moins à toute la zone Euro (Bruno Biancone, 1999).

Revue internationale P.M.E., vol. 14, $\mathrm{n}^{\text {os }} 3-4,2001$ 
Cette attention constante au marché italien n'entre d'ailleurs pas en contradiction avec l'évaluation positive de la position concurrentielle qu'ont les entreprises à forte croissance. Une part importante des entreprises appartenant à un groupe les situe parmi les cinq ou les dix principaux producteurs à l'échelle mondiale; les entreprises «exportatrices» sont également compétitives. Au contraire, la plupart des entreprises «dominées » et environ les trois quarts des «jeunes» ont une position plus faible, occupant au moins la vingtième position parmi les compétiteurs mondiaux. Le placement compétitif s'améliore lorsqu'on regarde l'arène européenne ou nationale.

La plupart des entreprises qui exportent et celles qui appartiennent à un groupe déclarent, en outre, avoir des attentes de stabilisation sur leurs propres marchés : quelques entreprises « jeunes » et « dominées » partagent cette évaluation. Au contraire, les entreprises « qui appartiennent à un groupe » et les entreprises «jeunes » voient leurs propres marchés croître. Le fait que les autres entreprises considèrent leurs propres marchés comme plutôt stables renforce l'hypothèse que leur croissance ne provient pas d'une augmentation externe de leur marché mais résulte plutôt d'une stratégie poursuivie consciemment par l'entrepreneur.

\subsubsection{Coopération entrepreneuriale}

Un deuxième élément qui caractérise les comportements différents des groupes d'entreprises se rapporte aux relations que les entreprises établissent avec les différents partenaires et les interlocuteurs avec qui elles sont constamment en contact (Bennet, 1998 ; Bennet et Robson, 1999).

\section{TABLEAU 5}

Importance attribuée aux différents partenaires aux quatre différents sous-ensembles d'entreprises en croissance

\begin{tabular}{lcccc}
\hline $\begin{array}{l}\text { Soutien à la croissance } \\
\text { offert par les partenaires }\end{array}$ & Jeunes & Exportatrices & $\begin{array}{c}\text { Appartenantes } \\
\text { à un groupe }\end{array}$ & Dominées \\
\hline Compétiteurs & 2,02 & 2,00 & 1,09 & 1,08 \\
Fournisseurs & 2,05 & $\mathbf{2 , 0 3}$ & $\mathbf{2 , 0 3}$ & $\mathbf{2 , 0 0}$ \\
Centres de recherche & 1,04 & 1,07 & 1,04 & 1,02 \\
Centres des services & 1,08 & 2,00 & 1,07 & 1,06 \\
Autres entreprises & 1,08 & 1,08 & $\mathbf{2 , 0 3}$ & $\mathbf{2 , 0 0}$ \\
Universités & 1,07 & 1,06 & 1,07 & 1,05 \\
\hline
\end{tabular}

Les scores représentent les valeurs moyennes assignées par les personnes interviewées : 1 = pas important ; 2 = peu important ; 3 = important ; 4 = très important. Les maximums de chaque ligne sont en italique, les maximums de chaque colonne sont en noir ( $1^{\mathrm{re}}$ et $2^{\mathrm{e}}$ priorités). 
Il faut d'abord mentionner ici que toutes les entreprises à forte croissance se caractérisent par un niveau considérable d'autosuffisance (vraie ou présumée), témoignée par les faibles scores que l'on attribue à tous les éléments relatifs aux rapports avec les tiers (voir tableau 5).

Seules les relations avec les fournisseurs - comme la documentation l'a fréquemment mis en évidence (Hull et Hjern, 1987; Taranto, 1990) - obtiennent les plus fortes évaluations. Seulement trois autres relations ressortent : le rôle des centres de services pour les entreprises «qui exportent»-qui témoignent de l'importance des activités d'accompagnement sur les marchés étrangers et du dégrèvement d'un certain nombre de tâches bureaucratiques - (Prencipe, 1997; Bennet et Robson, 1999); le rôle des autres entreprises chez celles qui appartiennent à un groupe - il est facile de voir ici l'importance du groupe ; le rôle des universités chez les entreprises « dominées », probablement lié aux besoins de trouver des solutions à certains problèmes techniques soulevés par des clients importants.

On relève, en outre, une certaine homogénéité d'évaluation en ce qui concerne les relations avec les conseillers. Les entreprises y ont recours plus ou moins fréquemment; cela dépend des groupes auxquels elles appartiennent, même si cette aide est en général considérée comme peu importante; il s'agit donc d'un besoin routinier et non stratégique.

Le recours à des accords avec d'autres entreprises (Pencarelli, 1995), qui touchent principalement la production et la commercialisation et, de façon marginale, la technologie, est plus varié. Ce sont les entreprises « jeunes » et celles «dominées » qui évidemment en font une plus grande utilisation.

Parmi les groupes d'entreprises «jeunes » et «exportatrices », ce sont les accords de commercialisation qui sont les plus importants, car ils permettent de faire des économies de distribution impossibles chez les entreprises qui appartiennent à un groupe. Pour les entreprises « dominées », les accords de production et de technologie sont particulièrement significatifs. Le groupe des entreprises qui exportent est, en revanche, moins intéressé à ces moyens.

\subsubsection{Rapport avec les institutions locales}

Le dernier élément des facteurs externes concerne le soutien apporté par les organismes, les institutions et les organisations locales ou nationales qui peuvent aider l'entreprise. L'évaluation moyenne de cette aide est généralement faible, avec un score qui dépasse difficilement 2.

L'institution qui semble avoir le plus de poids est la banque (Dellacasa, 1996), dépassée seulement par les associations d'entrepreneurs (Bennet, 1998). Pour tous les autres sujets considérés, les scores sont encore plus faibles et semblables : de la chambre de commerce en passant par les syndicats jusqu'aux administrations régionales.

Revue internationale P.M.E., vol. 14, $\mathrm{n}^{\text {os }}$ 3-4, 2001 
En ce qui a trait aux contenus de cette aide, il est évident que les aides reçues touchent principalement la production, auxquelles s'ajoutent, pour les entreprises « jeunes », les aides à la commercialisation et à l'exportation, les services pour la recherche et le recrutement. Parmi les entreprises qui exportent, l'aide porte sur la commercialisation et l'exportation, bien que l'évaluation de celle-ci soit faible. Le tableau 6 illustre l'importance de ces facteurs externes.

TABleau 6

Similitudes et spécificités des facteurs externes par groupes d'entreprises à forte croissance

\begin{tabular}{|c|c|c|}
\hline Entreprises & Marché & Relations extérieures \\
\hline Jeunes & $\begin{array}{l}\text { Fortement orientées vers le } \\
\text { marché italien. } \\
\text { Peu d'entreprises parmi les } \\
\text { premiers producteurs au } \\
\text { niveau mondial ou européen } \\
\text { (mais plus que parmi les } \\
\text { dominées). }\end{array}$ & $\begin{array}{l}\text { Les accords de commerce sont intenses } \\
\text { et les accords de production le sont moins. } \\
\text { L'aide principale provient des banques. } \\
\text { Le principal domaine d'intervention } \\
\text { des institutions est la production. } \\
\text { Les institutions locales fournissent } \\
\text { des informations. } \\
\text { L'utilisation des centres de services est très limitée. }\end{array}$ \\
\hline Exportatrices & $\begin{array}{l}\text { Fortement orientées vers les } \\
\text { marchés étrangers. } \\
\text { Ce groupe représente la part } \\
\text { la plus grande d'entreprises } \\
\text { qui se trouvent parmi les } \\
5 \text { ou } 10 \text { principaux } \\
\text { producteurs au niveau } \\
\text { européen. }\end{array}$ & $\begin{array}{l}\text { Il y a peu d'accords de commerce, mais les } \\
\text { accords de technologie sont plus intenses. } \\
\text { L'aide principale provient des banques. } \\
\text { Les principaux domaines d'intervention } \\
\text { sont la finance, le commerce et l'exportation. } \\
\text { Les institutions locales fournissent } \\
\text { une aide administrative. } \\
\text { L'utilisation des centres de services est limitée. }\end{array}$ \\
\hline $\begin{array}{l}\text { Appartenantes } \\
\text { à un groupe }\end{array}$ & $\begin{array}{l}\text { Fortement orientées vers } \\
\text { le marché italien. } \\
\text { Ce groupe a la part la plus } \\
\text { forte d'entreprises qui se } \\
\text { situent parmi les } 5 \text { ou } 10 \\
\text { principaux producteurs au } \\
\text { niveau mondial. }\end{array}$ & $\begin{array}{l}\text { Les accords commerciaux sont intenses } \\
\text { et il existe bon nombre d'accords de production. } \\
\text { L'aide principale provient des associations } \\
\text { d'entrepreneurs. } \\
\text { Les principaux domaines d'intervention des } \\
\text { institutions sont le financement et la production. } \\
\text { Les institutions locales fournissent informations } \\
\text { et assistance administrative. } \\
\text { L'utilisation de centres de services } \\
\text { est plutôt intense. }\end{array}$ \\
\hline Dominées & $\begin{array}{l}\text { Fortement orientées vers } \\
\text { le marché italien. } \\
\text { Ce groupe a la part la plus } \\
\text { forte d'entreprises qui se } \\
\text { situe au-delà de la } 20^{\mathrm{e}} \text { place, } \\
\text { soit sur le plan national ou } \\
\text { international. }\end{array}$ & $\begin{array}{l}\text { Il y a peu d'accords de commerce, } \\
\text { mais plusieurs accords de production. } \\
\text { L'aide principale provient des banques. } \\
\text { Les principaux domaines d'intervention des } \\
\text { institutions sont le financement et la production. } \\
\text { Les institutions locales fournissent informations } \\
\text { et assistance administrative. } \\
\text { L'utilisation des centres de services } \\
\text { est très intense. }\end{array}$ \\
\hline
\end{tabular}

Revue internationale P.M.E., vol. 14, $\mathrm{n}^{\text {os }} 3-4,2001$ 


\subsection{Les facteurs internes}

Un deuxième groupe de facteurs concerne chaque entreprise et les stratégies adoptées par l'entrepreneur (Corno, 1989; Marchini, 1995 a et b; Pencarelli, 1995). On peut classer parmi ces facteurs tous les éléments qui se rapportent aux choix stratégiques de l'entreprise et à l'utilisation de moyens formalisés de planification dans les choix à moyen terme (sous-section 5.2.1), l'organisation du travail, la gestion des ressources humaines et les canaux à travers lesquels on peut embaucher (sous-section 5.2.2), les trajectoires d'innovation suivies et les leviers financiers utilisés (sous-section 5.2.3). Les attitudes et les évaluations des entreprises pour chacun de ces facteurs ont été relevées à partir de plusieurs questions que l'on a reliées entre elles.

\subsubsection{Choix stratégiques}

Les facteurs ayant permis aux entreprises de croître (MEA, 1999 a et b) sont reliés aux différentes stratégies adoptées par les PME italiennes (Mussati, 1990; AA.VV., 1995 ; De Leo, 1995; Brunetti, Mussati et Corbetta, 1997; Corbetta, 2000), soit l'innovation, pour certaines d'entre elles, la flexibilité et l'adaptation aux besoins des clients et les prix bas pour d'autres.

Toutes les entreprises, par exemple, accordent une grande importance, pour leur propre croissance, à la qualité des produits et du service offert aux clients (voir tableau 7) bien que l'on relève certaines différences. Ainsi, pour les entreprises «jeunes », les prix bas et la nécessité de se faire connaître sur le marché et de consolider celui-ci revêtent une importance relativement grande, alors que les entreprises «dominées » sont, parmi les quatre groupes, celles qui croissent le plus avec la croissance du marché (liées à la croissance de leurs propres clients). Enfin, les entreprises qui « exportent » soulignent l'importance d'exploiter les marchés de niche et d'accorder une attention spéciale à la diffusion du produit.

Un deuxième élément important concerne la planification stratégique (Corno, 1989; AA.VV, 1995 ; Marchini, 1995b ; Arcari, 1999; Corbetta, 2000). Tout en se différenciant considérablement de la plupart des PME italiennes, la majorité des entreprises à forte croissance interviewées propose des stratégies à long terme, sauf les entreprises «dominées ». La présence d'un réel parcours d'élaboration des stratégies se manifeste par la formalisation des stratégies, même sous forme de documents écrits, et par la mise à jour systématique de celles-ci, parfois même chaque semestre.

Revue internationale P.M.E., vol. 14, nºs 3-4, 2001 
TABLEAU 7

Importance attribuée aux différents facteurs de croissance pour les quatre sous-ensembles d'entreprises à forte croissance

\begin{tabular}{lcccc}
\hline Leviers stratégiques & Jeunes & Exportatrices & $\begin{array}{c}\text { Appartenantes } \\
\text { à un groupe }\end{array}$ & Dominées \\
\hline Croissance du marché & 2,04 & 2,05 & 2,04 & 2,08 \\
Qualité élevée & $\mathbf{3 , 0 2}$ & $\mathbf{3 , 0 3}$ & $\mathbf{3 , 0 3}$ & $\mathbf{3 , 0 1}$ \\
Innovation & 2,07 & 2,06 & 2,03 & 2,02 \\
Prix bas & $\mathbf{3 , 0 2}$ & 2,08 & 3,00 & 2,09 \\
Produits de niche & 2,04 & 2,05 & 2,01 & 2,02 \\
Standardisation du produit & 2,00 & 2,03 & 2,01 & 2,00 \\
Services d'assistance & 3,00 & $\mathbf{3 , 0 3}$ & 3,02 & 2,09 \\
\hline
\end{tabular}

Les scores représentent les valeurs moyennes assignées par les personnes interviewées : 1 = pas important ; 2 = peu important ; 3 = important ; 4 = très important. Les maximums de chaque ligne sont en italique, les maximums de chaque colonne sont en noir ( $1^{\text {re }}$ et $2^{\mathrm{e}}$ priorités).

\subsubsection{Organisation du travail}

Parmi les inputs que l'entreprise recherche dans son secteur d'activité, on trouve une attention notable pour les variables touchant le capital humain et le capital financier (Dellacasa, 1996; Arcari, 1999).

L'organisation du travail est généralement de type hiérarchique dans la plupart des entreprises de tous les groupes. C'est un type d'organisation où l'entrepreneur délègue certains pouvoirs aux cadres d'entreprises qui sont contrôlés directement. Cette hiérarchisation est typique de l'organisation des PME italiennes où, en général, l'entrepreneur désire avoir sous son contrôle direct les fonctions à l'intérieur de l'entreprise (Corno, 1989; Preti, 1991; Corbetta, 2000). Un modèle d'organisation plus décentralisé (modèle à étoile) est relativement plus présent dans les entreprises «dominées » et dans les entreprises «qui appartiennent à un groupe».

À propos de la gestion des ressources humaines et particulièrement de la stabilité des relations de travail, le pourcentage élevé des employés engagés par un contrat temporaire indique deux choses : 1) une exigence de flexibilité et 2) une recherche de stabilité malgré la croissance de l'entreprise. Ainsi, on passe de $11 \%$ des employés temporaires dans les entreprises «jeunes », à $17 \%$ pour celles «qui exportent », à $19 \%$ pour celles «qui appartiennent à un groupe », pour atteindre $30 \%$ pour les entreprises «dominées ». Il est évident que cela s'explique dans ce dernier groupe par l'exigence de faire face aux pics de production liés à la concentration de la demande des clients peu nombreux dont les entreprises dépendent. Les entreprises « jeunes », au contraire, sont peut-être plus optimistes au regard de leurs perspectives de croissance et sont plus motivées à investir dans des ressources spécifiques qui se stabiliseront au fil du temps. 
Les employés sont surtout choisis selon leurs compétences en tant que spécialistes. Les entreprises qui exportent font exception, car elles considèrent comme relativement plus importante la capacité de travailler en groupe. Aux employés, on demande en outre d'être flexibles, surtout dans les entreprises «jeunes » et dans celles « dominées ». L'organisation du travail la plus couramment observée prévoit l'assignation de tâches spécifiques à chaque employé; tandis que pour la plupart des entreprises «qui appartiennent à un groupe », les employés doivent pouvoir remplir plusieurs fonctions.

Le recrutement de jeunes à leur première expérience de travail est l'option la plus retenue par tous les groupes, même si l'engagement de personnel déjà formé reste significatif. D'un côté, la difficulté de trouver du personnel déjà formé peut expliquer le recours aux jeunes et aux employés venant des concurrents; de l'autre, on semble gagner à engager des nouveaux, de façon à former du personnel selon la philosophie de l'entreprise. Les entreprises à forte croissance sont, en effet, des entreprises où l'organisation et les rapports avec les clients comptent beaucoup (Karlsson, Johannisson et Storey, 1993 ; Klofsten, Jones-Evans et Lindell, 1997) et elles ont besoin de relations internes marquées par l'auto-apprentissage et la résolution de problèmes.

Le recrutement de cadres d'entreprise pour accompagner les processus de croissance est aussi un moyen significatif (Preti, 2001). La plus haute évaluation à cet égard est notamment exprimée par les entreprises « jeunes » qui accordent beaucoup d'importance stratégique à cette ressource.

\subsubsection{Parcours d'innovation}

Un troisième élément clé concerne l'innovation qui explique dans une large mesure la forte croissance. Lorsque l'innovation est de type économie du travail, tout en agissant sur la productivité et sur les processus, un taux élevé d'innovation n'implique pas nécessairement une augmentation de l'emploi ${ }^{15}$.

Le nombre moyen d'innovations produites par chaque entreprise (calculé par rapport au nombre d'entreprises qui appartiennent au groupe) montre que les entreprises «qui exportent» forment le groupe le plus innovateur, probablement parce qu'elles sont stimulées par la forte concurrence des marchés internationaux. Ensuite, on trouve les entreprises « qui appartiennent à un groupe » pour ce qui est

15. Tout en ne voulant pas nous engager dans un long débat sur les indicateurs les plus appropriés pour mesurer le niveau d'innovation d'une entreprise, il est nécessaire de signaler que les informations rassemblées étaient basées sur l'auto-évaluation - de l'entrepreneur interviewé - en ce qui concerne le nombre des nouveaux produits ou procédés qui ont été introduits ou modifiés au cours des trois dernières années.

Revue internationale P.M.E., vol. 14, $\mathrm{n}^{\text {os }} 3-4,2001$ 
des innovations de produit et celles « dominées » pour les modifications de produit et les innovations de procédés (ce qui représente vraiment leur spécialité, étant donné qu'elles fabriquent des produits intermédiaires pour leurs clients industriels).

D'autres informations révèlent que l'utilisation de brevets est modeste, mais pas inexistante parmi les entreprises à forte croissance. Le groupe qui réunit la plupart des entreprises détentrices de brevets est celui des entreprises «qui exportent ». Les entreprises «dominées », tout en présentant un bon nombre d'innovations de produits et de procédés, détiennent beaucoup moins de brevets que sur le plan national.

La plupart des entreprises n'ont pas encore intégré de fonction de R-D ; cependant, l'investissement dans ce domaine par quelques entreprises est important. Parmi celles « qui appartiennent à un groupe », la présence de R-D à l'interne ou de ressources humaines dédiées à cette fonction est significative.

Enfin, en ce qui concerne les sources de financement, le comportement des entreprises de l'échantillon semble correspondre au comportement général des entreprises italiennes. Grâce aux données de la Centrale de budgets (Centrale dei Bilanci, 2000), nous savons qu'entre 1990 et 1994 la croissance de la marge brute a permis aux entreprises d'augmenter leur autofinancement. En même temps, les entreprises de plus grande taille ont recours aux marchés financiers pour améliorer la structure de leurs propres passifs. C'est connu, les petites entreprises éprouvent souvent des difficultés à accéder au capital de risque et quelquefois aussi au crédit à long terme (voir Dellacasa, 1996).

Les sources de financement que les entreprises à forte croissance utilisent principalement sont les bénéfices non distribués, les investissements directs des entrepreneurs et le levier de la dette à court terme (voir le tableau 8). L'émission d'actions ou d'obligations, l'entrée de nouveaux actionnaires et même l'utilisation de fonds publics sont décidément moins fréquemment observées (voir Corbetta et Dubini, 1997).

Dans les entreprises « jeunes », les fonds propres de l'entrepreneur représente la solution la plus courante, suivie par le recours aux bénéfices non distribués; cela va de pair avec les difficultés du système financier italien à soutenir des entrepreneurs et des organisations « patrimoniales » qui ne disposent pas d'une couverture convenable de garanties réelles dans les processus de croissance. Comme on devait s'y attendre, l'entrée de nouveaux actionnaires n'est pas significative ; l'entreprise, créée depuis peu, doit encore s'organiser avant d'élargir sa propriété. Le faible recours aux financements publics, qui pourraient représenter une solution avantageuse pour les entreprises qui peuvent difficilement avoir accès aux marchés des capitaux, est moins évident (Brunetti, Mussati et Corbetta, 1997). 
TABLEAU 8

Importance des différentes sources de financement pour les quatre sous-ensembles d'entreprises à forte croissance

\begin{tabular}{lcccc}
\hline Leviers stratégiques & Jeunes & Exportatrices & $\begin{array}{c}\text { Appartenantes } \\
\text { à un groupe }\end{array}$ & Dominées \\
\hline Profits non distribués & 2,05 & $\mathbf{3 , 0 0}$ & 2,06 & 2,08 \\
Fonds propres & $\mathbf{3 , 0 5}$ & 2,08 & $\mathbf{2 , 0 9}$ & $\mathbf{2 , 0 9}$ \\
Nouveaux actionnaires & 1,02 & 1,07 & 1,04 & 1,03 \\
Émissions d'actions & & & & \\
$\quad$ ou d'obligations & 1,02 & 1,04 & 1,01 & 1,01 \\
Endettement à court terme & 2,00 & 2,08 & 2,02 & 2,04 \\
Endettement à long terme & 2,03 & 2,05 & 2,05 & 2,03 \\
Fonds publics & 1,06 & 1,08 & 2,01 & 1,07 \\
\hline
\end{tabular}

Les scores représentent les valeurs moyennes assignées par les interviewés : 1 = non important; $2=$ peu important $; 3=$ important $; 4=$ très important. Les maximums de chaque ligne sont en italique, les maximums de chaque colonne sont en noir ( $1^{\text {re }}$ et $2^{\mathrm{e}}$ priorités).

Dans le cas des entreprises «qui exportent», outre l'autofinancement, le recours à l'endettement à court terme qui, surtout auparavant, était justifié par l'exigence d'opérer au comptant sans autres moyens plus raffinés pour couvrir des risques de change a une importance relative.

Parmi les entreprises «qui appartiennent à des groupes », l'utilisation du financement public est plus grande. Cela signale qu'au sein du groupe il y a une expertise quant à la manière de gérer les affaires et d'adresser les demandes, éventuellement combinée à une meilleure circulation de l'information.

Enfin, les entreprises «dominées » semblent être conservatrices dans l'utilisation de levier financier. Elles exploitent, outre l'autofinancement, seulement l'endettement à court terme et, de façon moins importante, l'endettement à long terme. Les principales différences dans l'articulation de ce deuxième groupe de facteurs internes sont présentées au tableau 9.

\subsection{Les motifs de la croissance}

Il existe une longue tradition de recherches portant sur les motivations psychologique et sociologique des entrepreneurs - tels les besoins d'accomplissement et de contrôle (Chell, 1986; Stanworth et al., 1989; Mussati, 1990 ; Marchini 1995a) et qui lie les motivations des entrepreneurs au besoin de succès, au désir de responsabilité, à l'attitude à l'égard du risque, à l'orientation vers le futur, aux habiletés d'organisation et autres choses (Corno, 1989). 
TABLEAU 9

Similitudes et spécificités des facteurs internes, selon les groupes d'entreprises à forte croissance

\begin{tabular}{|c|c|c|c|}
\hline Entreprises & Organisation du travail & Financement & Innovation \\
\hline Jeunes & $\begin{array}{l}\text { Peu de contrats à temps déterminé. } \\
\text { Des nouveaux employés, on exige } \\
\text { de la flexibilité et une compétence } \\
\text { de spécialiste. } \\
\text { Adoption intense de primes pour chaque } \\
\text { employé ( } 62 \% \text { des entreprises). } \\
\text { Utilisation intensive de groupes } \\
\text { d'employés. }\end{array}$ & $\begin{array}{l}\text { Le maximum de } \\
\text { recours aux fonds } \\
\text { propres. } \\
\text { Moins de recours à } \\
\text { l'endettement } \\
\text { bancaire (à court } \\
\text { et à long terme). }\end{array}$ & $\begin{array}{l}\text { Peu } \\
\text { innovatrices. }\end{array}$ \\
\hline Exportatrices & $\begin{array}{l}\text { Peu de contrats à temps déterminé. } \\
\text { Des nouveaux employés, on exige } \\
\text { de la flexibilité et une compétence } \\
\text { de spécialiste. } \\
\text { On exige de la flexibilité de tous les } \\
\text { employés (environ } 65 \% \text { des entreprises). } \\
\text { Adoption intense de primes pour chaque } \\
\text { employé (58\% des entreprises). } \\
\text { Peu de groupes d'employés. }\end{array}$ & $\begin{array}{l}\text { Le maximum de } \\
\text { recours aux } \\
\text { bénéfices non } \\
\text { distribués. } \\
\text { Recours significatif } \\
\text { aux fonds propres } \\
\text { et à l'endettement } \\
\text { bancaire (à court } \\
\text { et à long terme). }\end{array}$ & $\begin{array}{l}\text { Les plus } \\
\text { innovatrices. }\end{array}$ \\
\hline $\begin{array}{l}\text { Appartenantes } \\
\text { à un groupe }\end{array}$ & $\begin{array}{l}\text { Peu de contrats à temps déterminé. } \\
\text { Des nouveaux employés, on exige } \\
\text { une compétence de spécialiste. } \\
\text { On exige de la flexibilité de tous les } \\
\text { employés (environ } 47 \% \text { des entreprises). } \\
\text { Peu de primes pour chaque employé } \\
\text { (environ } 47 \% \text { des entreprises). } \\
\text { Peu de groupes d'employés. }\end{array}$ & $\begin{array}{l}\text { Recours significatifs } \\
\text { aux fonds propres. } \\
\text { Recours aux fonds } \\
\text { propres et à } \\
\text { l'endettement } \\
\text { bancaire (à court } \\
\text { et à long terme). }\end{array}$ & $\begin{array}{l}\text { Plutôt } \\
\text { innovatrices. }\end{array}$ \\
\hline Dominées & $\begin{array}{l}\text { Beaucoup de contrats à temps déterminé. } \\
\text { Des nouveaux employés, on exige } \\
\text { de l'expérience et des compétences } \\
\text { de spécialiste. } \\
50 \% \text { des entreprises exigent de la } \\
\text { flexibilité de tous les employés et } \\
29 \% \text { l'exigent seulement des employés } \\
\text { qui ont des responsabilités. } \\
\text { Peu de primes pour chaque employé } \\
\text { (environ } 45 \% \text { des entreprises). } \\
\text { Utilisation intensive de groupes } \\
\text { d'employés. }\end{array}$ & $\begin{array}{l}\text { Recours significatifs } \\
\text { aux fonds propres } \\
\text { et aux bénéfices } \\
\text { non distribués. }\end{array}$ & $\begin{array}{l}\text { Les moins } \\
\text { innovatrices. }\end{array}$ \\
\hline
\end{tabular}

Les processus de croissance des entreprises, qui se déroulent par phases successives, du démarrage au décollage de l'entreprise (Churchill et Lewis, 1983 ; Scott et Bruce, 1987; Arcari, 1999), proviennent, du point de vue des motifs, amplement de la même source. Il devient ainsi important de s'interroger sur l'attitude à l'égard des risques et de la croissance en contexte italien (Mussati, 1990; Biggiero 
et Caroli, 1995 ; Marchini, 1995b). La plupart des répondants pensent que la croissance est un signe que l'entreprise prospère. Ce n'est que dans le cas des entreprises « dominées » que le pourcentage de celles qui considèrent la croissance comme une preuve de réussite est inférieur à $50 \%$. La croissance serait donc une "étape nécessaire pour survivre » pour $27 \%$ des entreprises « dominées » et $21 \%$ pour les « jeunes ». Dans le premier cas, la croissance est un signe indirect - ajouté à la part des entreprises qui répondent que c'est «quelque chose de très difficile »-d'une croissance imposée plutôt que désirée. Les entreprises «jeunes», au contraire, doivent effectivement atteindre une dimension optimale pour pouvoir survivre, tout en considérant la croissance comme un défi.

La croissance comporte, du reste, des éléments de risque (Curran et Blackburn, 1991 ; Karlsson, Johannisson et Storey, 1993), ce qui semble préoccuper le plus les entrepreneurs qui songent à l'instabilité de la demande, avec des scores toujours supérieurs à 2,5. Les différences entre les quatre groupes d'entreprises, bien que minimes, sont éclairantes : les entreprises «qui exportent » perçoivent un risque plus élevé dans l'engagement de nouveaux employés (avec la possibilité accrue que les marchés étrangers soient l'objet d'un choc exogène d'une grande portée); en revanche, les entreprises «qui appartiennent à un groupe » ne courent évidemment pas des risques d'ordre financier, car elles profitent du soutien du groupe.

\section{Quelques explications sur la croissance des PME italiennes}

L'échantillon analysé fournit beaucoup plus d'indices mettant en relief l'hétérogénéité des trajectoires de croissance que les éléments communs d'interprétation. La croissance des PME dépend d'un ensemble très particulier de facteurs internes, externes et de motivations. C'est l'entrepreneur qui, en effet, adopte une stratégie pour rester sur le marché et affronter, de façon active, la concurrence. La croissance fait généralement partie de cette stratégie, même si elle n'est pas l'objectif final ; parfois, la croissance représente le moyen pour atteindre ce dernier, évidemment en tenant compte de l'analyse subjective des risques associés à cette croissance.

Même au cours de l'enquête - en présence de performances de croissance parfois extraordinaires - d'autres comportements de long terme sont ressortis, tels que la nécessité de cumuler moyens financiers convenables et de développer des normes de fonctionnement rigoureuses (touchant l'organisation et les coûts) qui ne permettent pas de voir la forte croissance comme quelque chose de temporaire.

Cela s'explique par les objectifs à long terme qui guident la planification et orientent le processus de croissance. Par conséquent, même si la documentation a clairement identifié les différents facteurs qui favorisent ou freinent la croissance (Hull et Hjern, 1987; Brunetti, Mussati et Corbetta, 1997), leur existence ne représente jamais une condition nécessaire et suffisante pour réaliser effectivement ces trajectoires.

Revue internationale P.M.E., vol. 14, $\mathrm{n}^{\text {os }} 3-4,2001$ 
Il s'ensuit donc que la décision de grandir - qui résulte de différentes stratégies visant les coûts et les objectifs - représente une forte spécificité ; cet élément varie de façon remarquable chez les entreprises, tout en étant influencé soit par la localisation géographique, soit par le type de secteur, soit, enfin, par la taille de l'entreprise. Les PME à forte croissance de l'échantillon offrent une telle spécificité tout en ayant des éléments communs qui permettent de les regrouper dans quatre catégories et que l'on a résumées au tableau 10.

TABLEAU 10

Les caractéristiques des trajectoires et des motivations à la croissance

\begin{tabular}{|c|c|}
\hline Groupe & Description \\
\hline Jeunes & $\begin{array}{l}\text { Ces entreprises, dont la création repose sur une idée maîtresse innovatrice, sont équi- } \\
\text { pées pour satisfaire aux exigences changeantes des marchés. Les ressources straté- } \\
\text { giques sont le savoir-faire et le zèle de chaque employé. Tout en s'orientant fortement } \\
\text { vers le marché national, elles n'ont pas (encore) de position de leadership sur le plan } \\
\text { international. Elles ont suivi des trajectoires de croissance non linéaires, financées } \\
\text { principalement par les ressources de l'entrepreneur. Elles se sont révélées vraiment } \\
\text { actives dans l'utilisation des accords commerciaux et productifs et elles ont profité } \\
\text { de l'aide d'institutions publiques locales, surtout pour obtenir de l'information. }\end{array}$ \\
\hline Exportatrices & $\begin{array}{l}\text { Ayant une bonne / très bonne position concurrentielle, elles visent essentiellement à } \\
\text { répondre aux rapides changements de marché, tout en maintenant une organisation } \\
\text { interne solide. Elles ont suivi des trajectoires de croissance relativement linéaires, } \\
\text { qui ont été principalement financés par les profits non distribués. Elles sont plutôt } \\
\text { innovatrices et recourent aux accords technologiques (plus que commerciaux). Elles } \\
\text { ont profité de l'aide publique, surtout en ce qui concerne les financements et les } \\
\text { procédures administratives. }\end{array}$ \\
\hline $\begin{array}{l}\text { Appartenantes } \\
\text { à un groupe }\end{array}$ & $\begin{array}{l}\text { Tout en ayant une flexibilité plus faible, elles jouissent d'économies externes spéci- } \\
\text { fiques dont elles savent tirer parti. Les ressources stratégiques s'appuient sur un bon } \\
\text { niveau d'innovation et une structure de gestion solide. Elles ont suivi des trajectoires } \\
\text { de croissance fondamentalement linéaires, financées pour la plupart par les fonds } \\
\text { propres ou par le groupe. Elles ont aussi recours à des consultants, comme aide } \\
\text { à la production, s'appuyant aussi sur les centres de services et les associations } \\
\text { d'entrepreneurs. }\end{array}$ \\
\hline Dominées & $\begin{array}{l}\text { Ces entreprises sont caractérisées par une faculté élevée d'adaptation et de } \\
\text { personnalisation des produits. Leurs ressources stratégiques sont le capital humain doté } \\
\text { d'une bonne capacité de savoir-faire et de résolution de problèmes. Elles ont suivi des } \\
\text { trajectoires de croissance totalement financées par elles-mêmes (fonds propres et pro- } \\
\text { fits non distribués). Elles se limitent au marché national où elles se positionnent bien } \\
\text { sur le plan de la compétition. Elles concluent fréquemment des accords de production } \\
\text { avec d'autres entreprises et reçoivent l'aide des banques et des centres de services. }\end{array}$ \\
\hline
\end{tabular}

Si l'on revient aux trois types de facteurs de la croissance (externes, internes et motivations personnelles), il est possible de classer qualitativement (en attribuant un score de 1 à 14) les éléments qui caractérisent chacun de ces facteurs pour obtenir un score récapitulatif de leur importance relative sur la croissance pour ces quatre groupes d'entreprises. 
Le score synthétique le plus élevé se trouve chez les entreprises « qui appartiennent à un groupe ». Le groupe a donc pour rôle d'accélérer le développement, tout en offrant des externalités privées importantes pour la croissance. Comme nous l'avons déjà mentionné, il s'agit surtout des externalités touchant la finance (il faut financer les processus de croissance) et la commercialisation.

Les entreprises « jeunes » arrivent au deuxième rang au regard du processus de croissance, tout en étant très centrées sur les facteurs internes. En leur faveur, on doit ajouter une autre motivation : à mesure que le processus de croissance se consolide, les entreprises un peu moins jeunes profitent aussi de facteurs externes qui permettent d'activer ultérieurement la croissance.

Les entreprises «dominées », enfin, ont une position plus faible à laquelle contribue le rôle le plus faible des facteurs de motivation. La seule solution pour continuer à croître consiste à favoriser la diversification pour éventuellement sortir de cette catégorie.

TABLEAU 11

Une évaluation synthétique de la contribution à la croissance des différents facteurs

\begin{tabular}{lccccc}
\hline $\begin{array}{l}\text { Facteurs } \\
\text { de croissance }\end{array}$ & Jeunes & Exportatrices & $\begin{array}{c}\text { Appartenantes } \\
\text { à un groupe }\end{array}$ & Dominées & $\begin{array}{c}\text { Moyenne } \\
\text { des groupes }\end{array}$ \\
\hline Externes $^{\mathrm{a}}$ & 179 & 357 & 571 & 286 & 348 \\
Internes $^{\mathrm{b}}$ & 800 & 533 & 533 & 150 & 504 \\
Motivation $^{\mathrm{c}}$ & 500 & 333 & 667 & 0 & 375 \\
Moyenne des facteurs & 493 & 408 & 590 & 146 & \\
\hline
\end{tabular}

Les scores sont relatifs et non pas absolus et varient entre 0 et 1000 .

a. Les éléments sur lesquels est basée l'évaluation synthétique des facteurs externes sont les suivants : l'ouverture vers l'étranger, le leadership sur le marché, les accords commerciaux, les accords de production, les consultations extérieures, le rôle des institutions et les centres de services.

b. Les éléments sur lesquels est basée l'évaluation synthétique des facteurs internes sont les suivants : la stabilité des employés, les primes salariales, le travail en groupe, la capacité d'innovation, la planification stratégique.

c. Les éléments sur lesquels est basée l'évaluation synthétique des facteurs de motivation sont les suivants : l'attitude à l'égard du risque et de la croissance.

Les PME italiennes à forte croissance se montrent, généralement, centrées sur elles-mêmes, comme l'indique le score élevé attribué aux facteurs internes (voir tableau 11). Ainsi, nous avons affaire à des entreprises peu contrôlées par les entrepreneurs, concurrentielles sur leurs propres marchés, discrètement innovatrices, ayant une forte tendance à personnaliser les productions et les services, habituées à s'appuyer sur une stratégie à moyen et long terme, tout en planifiant leurs propres phases de croissance.

Revue internationale P.M.E., vol. 14, nºs 3-4, 2001 
Ces entreprises doivent beaucoup à leur milieu. On pourrait aussi considérer leur attitude de repli sur elles-mêmes, comme une conséquence d'une évaluation négative de la qualité des biens locaux mis à leur disposition; cette évaluation est toutefois trop sévère. Il vaudrait mieux supposer que, dans leur effort pour soutenir leur croissance, ces entreprises doivent nécessairement concentrer leur attention et leurs énergies à l'interne pour ensuite se consolider, s'ouvrir à l'externe pour exploiter toutes les synergies que peut susciter l'interaction avec le milieu où elles ont été créées et où elles œuvrent.

\section{Les politiques}

En conclusion et à la suite des entrevues avec les interlocuteurs privilégiés que nous avons pu joindre, nous avons pu mener une réflexion approfondie sur les politiques industrielles qui concernent les PME (Bramanti, Scarpinato et Mussati, 2000) pour vérifier - avec des résultats attendus, (CER-IRS, 2000; Brancati, 2001) mais qui sont également décourageants - si les « gazelles » peuvent bénéficier de ces politiques tout au long de leur parcours de croissance et comment elles procèdent.

Avant tout, il faut dire qu'il est impossible de déceler un lien, même faible, entre les trajectoires de croissance des PME à forte croissance et le recours aux politiques industrielles à leur disposition. Les entreprises croissent et se développent grâce à leur propre capacité de compétition, à l'originalité de leur idée de départ, à l'accumulation et au renforcement de compétences spécifiques distinctives et à leur stratégie de croissance. Mais, à l'occasion, il arrive aussi que des entreprises recourent à des programmes d'aide clairs, simples, faciles à appliquer en temps opportun.

Les programmes législatifs que nous avons analysés - composés de 16 normes nationales amplement diffusées - sont non seulement peu utilisés par les entreprises à forte croissance, mais, dans certains cas, ils sont également peu connus ; ce qui confirme les analyses précédentes (Dellacasa, 1996; Corbetta et Dubini, 1997). Seulement trois interventions législatives de type automatique sont très bien connues par $60 \%$ des interviewés : il s'agit de la Loi 1329/65 (Sabatini) ${ }^{16}$, de la Loi 488/92 (interventions extraordinaires pour le Midi) ${ }^{17}$ et de la Loi 489/94 (Loi Tremonti) ${ }^{18}$.

16 Loi 1329/65 : mesures pour acheter de nouveaux équipements. La loi réglemente les modalités de rédaction d'un contrat d'achat et de vente d'équipement. Ceux-ci, bien spécifiés, font l'objet de garantie des effets émis (effets à cinq ans de date).

17. Loi 488/92 : politique structurée d'intervention extraordinaire pour le Midi. La loi prévoit des facilités en termes de cotisations à fonds perdu pour les investissements et le leasing (mobilier et immobilier) rendus plus faciles pour les PME implantées dans les régions méridionales.

18. Loi 489/94 : dispositions fiscales urgentes pour accélérer la reprise de l'économie et de l'occupation, ainsi que pour réduire la charge du contribuable (Loi Tremonti). Cette loi vise à favoriser la naissance et le développement de nouvelles initiatives entrepre- 
La connaissance d'un programme ne signifie pas que les entreprises y recourent souvent; cela confirme deux types de considérations déjà évoquées dans des études sur ce sujet (Mussati, 1990; Brunetti, Mussati et Corbetta, 1997; Bramanti et Scarpinato, 1999) :

a) d'une part, un système d'encouragement extrêmement fragmentaire et se recoupant souvent, comme c'est le cas actuellement, pousse les entreprises à privilégier certains programmes seulement ;

b) d'autre part, les règles d'entrée et les modalités d'opérationnalisation des programmes d'encouragement peuvent constituer, dans plusieurs cas, un «obstacle» à leur utilisation.

Le fait que les mesures les plus appréciées par les entreprises soient les plus anciennes, dont le fonctionnement est désormais connu, ou celles ayant un large impact, peu sélectives et dont les procédures se ressemblent, n'est pas un hasard. À cet égard, beaucoup d'entreprises ont signalé leur intérêt pour des moyens de type automatique, comme la loi Tremonti, qui est la plus connue.

L'une des raisons du désintérêt des entreprises à forte croissance à l'égard des mesures de politique industrielle est, en effet, la considérable charge bureaucratique (en termes de nombre de documents et de complexité des formulaires) qu'il faut assumer pour présenter une demande dont la réponse est toujours incertaine et le plus souvent différée. Cette lourdeur suffit le plus souvent à décourager les firmes ciblées de présenter leur demande. Lorsque des entreprises montrent une appréciation à l'égard d'un programme, il s'agit souvent d'entreprises qui ont recours à des conseillers pour présenter et accompagner la demande.

À partir des opinions émises par les interlocuteurs interviewés au sujet des problèmes que posent les programmes actuellement offerts aux entreprises, nous pouvons dégager certaines pistes de solution (voir tableau 12). Même si certaines mesures ont été prises pour assouplir les procédures et simplifier l'accès aux programmes, au dire de tous les interlocuteurs, il reste beaucoup à faire. À tout cela il faudrait, en outre, adjoindre un parcours des procédures beaucoup plus clair touchant le temps pour évaluer les demandes et, pareillement des garanties à l'égard de l'existence de fonds au moment où l'on présente la demande. La réorganisation des normes par zones de compétence où pourraient arriver les demandes constitue une autre exigence (Brancati, 2001).

neuriales, surtout celles qui ont été mises sur pied par des jeunes de moins de 32 ans. Parmi les différentes mesures prévues par la loi, soulignons l'absence de taxation sur le revenu d'entreprise réinvesti $(50 \%$ du total des investissements faits dans la période examinée est exclu de l'impôt des entreprises); la réduction de l'impôt des personnes juridiques pour les sociétés cotées est de $16 \%$ si le patrimoine net ne dépasse pas les 500 milliards de lires (mesure en vigueur jusqu'au 31 décembre 1997); la simplification des procédures et la réduction des sanctions pour des irrégularités de forme.

Revue internationale P.M.E., vol. 14, nºs 3-4, 2001 
TABLEAU 12

Problématiques et solutions évoquées par les répondants au sujet des programmes d'encouragement

\begin{tabular}{ll}
\hline Problèmes & Solutions \\
\hline Bureaucratisation, complexité des procédures. & $\begin{array}{l}\text { Amélioration des structures assurant la gestion } \\
\text { des encouragements / incitations. Collaboration } \\
\text { avec des associations professionnelles et } \\
\text { industrielles. }\end{array}$ \\
\hline $\begin{array}{l}\text { Hasard, discontinuité, retards dans l'utilisation } \\
\text { des moyens disponibles. Retard dans la mise } \\
\text { en œuvre de lois / mesures d'encouragement. }\end{array}$ & $\begin{array}{l}\text { Contrôle permanent de l'état d'application et } \\
\text { d'utilisation des lois / mesures d'encouragement. }\end{array}$ \\
\hline $\begin{array}{l}\text { Lois / mesures d'encouragement qui se limitent } \\
\text { aux seules entreprises qui en ont le plus besoin. }\end{array}$ & $\begin{array}{l}\text { Définition plus claire des critères de sélection; } \\
\text { évaluation plus attentive des conditions } \\
\text { d'admissibilité ; plus grande utilisation } \\
\text { de mécanismes « de rotation ». }\end{array}$ \\
\hline
\end{tabular}

Un système permanent de contrôle de mise en œuvre des normes et de financements plus facilement accessibles pourrait résumer la proposition finale; en particulier, il s'agirait:

a) de recueillir et d'uniformiser les informations ;

b) d'évaluer la capacité de réponse en région;

c) d'identifier les problèmes particuliers et les obstacles à l'accès aux fonds ;

d) d'inciter les autorités à instaurer des normes plus souples pour accroître l'efficacité des interventions ;

e) de mieux faire connaître les possibilités offertes pour les entreprises ;

f) d'améliorer éventuellement la formation du personnel pour soutenir les entreprises lors du processus de présentation des demandes.

La gestion opérationnelle de ce système exige la participation de tous les acteurs publics et privés qui s'occupent déjà aujourd'hui d'accompagner les entreprises, tout en faisant converger sur ces programmes toute une série de ressources qui déjà s'occupent ailleurs d'autres programmes (ou de familles de programmes) d'encouragement.

S'il est vrai que les «gazelles » montrent qu'elles n'ont pas eu besoin de ces aides pour réaliser leur croissance, on peut souhaiter qu'un système, fonctionnel et incitatif, élimine certains aspects qui empêchent d'autres PME de s'engager dans un processus de croissance, tout en augmentant le nombre des entreprises susceptibles de créer de l'emploi. 


\section{Bibliographie}

AA.VV. (1995), Il libro della piccola impresa, Roma, Adnkronos Libri.

AnAstasia, B. et G. CORÒ (1996), Evoluzione di un' economia regionale. Il Nordest dopo il successo, Portogruaro, Ediciclo.

ARCARI, A. (1999), a cura di, I processi di crescita delle PMI, Milan, Egea.

Audretsch, D. (1995), Innovation and Industry Evolution, Cambridge, MIT Press.

Banco Ambrosiano Veneto (dir.) (1996), Nord-Est : fattori di competitività, Bologna, Il Mulino.

BENNET, R.J. (1998), «Business associations and their potential contribution to the competitiveness of SMEs », Entrepreneurship \& Regional Development, vol. 10, no 3, p. 243-260.

BENNET, R.J. et J.A. RoBSON (1999), «The use of external business services by SMEs in Britain », Entrepreneurship \& Regional Development, vol. 11, n 2, p. 155-180.

Biggiero, L. et M. CAROLI (1995), La mortalità industriale. Aspetti teorici e risultati empirici, Milano, Guerini Studio.

BIRCH, D. (1987), Job Creation in America : How Our Smallest Companies Put the Most People to Work, New York, Free Press.

Bramanti, A. et M. SCARPINAto (1999), Politiche e interventi della CEE e degli stati membri per l'artigianato : analisi e confronti. Il caso Italia, Gruppo CLAS Artigianfin Progetti SpA. Milano e Roma, Rapporto di ricerca.

Bramanti, A., M. Scarpinato et G. Mussati (2000), "High-growth SMEs and employment. The Italian case », Part III - Best Practices Policies : An Assessment, Milan, Università Bocconi, CERTeT.

Bramanti, A. et L. SenN (1992), «Struttura ed evoluzione dell'economia padana», AA.VV., La Padania, una regione italiana in Europa, Torino, Fondazione Giovanni Agnelli, p. 15-120.

BRANCATI, R. (dir.) (2001), Analisi e metodologie per la valutazione delle politiche industriali, Milan, Franco Angeli.

Brock, W. et D. Evans (1986), The Economics of Small Business, New York, Holmer \& Meier.

Brunetti, G., G. Mussati et G. Corbetta (dir.) (1997), Piccole e medie imprese e politiche di facilitazione, Milan, Egea.

BRUNO BIANCONE, A.M. (1999), L'impresa minore nel processo di internazionalizzazione dei mercati. Sviluppo dimensionale e strategie competitive, Torino, Giappichelli.

CENTRAlE DEI BILANCI (2000), Economia e finanza delle imprese italiane, Tredicesimo Rapporto 1982-1998, Rome, Bancaria Editrice.

CER-IRS (2000), Fra decentramento amministrativo e integrazione europea, Decimo rapporto CER-IRS sull'industria e la politica industriale italiana, Bologna, Il Mulino.

CHELl, E. (1986), «The entrepreneurial personality: a review and some theoretical developments », J. Curran, J. Stanworth et D. Watkins (dir.), The Survival of the Small Firm : The Economics of Survival and Entrepreneurship, vol. 1. Cambridge, Gower, p. 102-119.

Revue internationale P.M.E., vol. 14, nos 3-4, 2001

(C) 2002 - Presses de l'Université du Québec

Édifice Le Delta I, 2875, boul. Laurier, bureau 450, Sainte-Foy, Québec G1V 2M2 • Tél. : (418) 657-4399 - www.puq.uquebec.ca

Tiré de : Revue internationale P.M.E., vol. 14, nos 3-4, sous la direction de Pierre-André Julien. 
ChurChill, N.C. et V.L. Lewis (1983), «The five stages of small business growth», Harvard Business Reviews, no 3, p. 30-50.

Corbetta, G. (2000), Le medie imprese. Alla ricerca della loro identità, Milan, Egea.

CORBETTA, G. et P. DUBINI (1997), «Le politiche di facilitazione allo sviluppo delle PMI : i risultati di un'indagine quantitativa », G. Brunetti, G. Mussati et G. Corbetta, (dir.), Piccole e medie imprese e politiche di facilitazione, Milan, Egea, p. 53-67.

CORNO, F. (1989), Lo sviluppo del sapere imprenditoriale nel governo dell' impresa, Milan, Egea.

CURRAn, J. et R.A. BLACKBURN (dir.) (1991), Paths of Enterprise. The Future of the Small Business, Londres, Routledge.

CURRAn, J., J. StANWORTH et D. WATKIns (dir.) (1986a), The Survival of the Small Firm. The Economics of Survival and Entrepreneurship, vol. 1, Cambridge, Gower.

CURRAn, J., J. STANWORTH et D. WATKIns (dir.) (1986b), The Survival of the Small Firm. Employment, Growth, Technology and Politics, vol. 2, Cambridge, Gower.

DE LEO, F. (1995), Le determinanti del vantaggio competitivo. Il contributo della ResourceBased View, Milan, Egea.

Dellacasa, G. (1996), a cura di, Economia delle piccole e medie imprese. Aspetti finanziari, Milan, Franco Angeli.

DePARTMENT of TRADE AND INdustry (DTI) (1996), Small Firms in Britain, Londres.

EFER (1996), Europes's 500 Dynamic Entrepreneurs. The Job Creators, Bruxelles, Diegem.

GALLI, G. (1999), I processi di internazionalizzazione delle piccole e medie imprese : analisi dei percorsi di sviluppo nel settore meccano-ceramico, Torino, Giappichelli.

Golfetto, F. (1988), Dimensioni strutturali del sistema industriale italiano, Milan, Egea.

HARHOFF, D., K. StAHL et M. Woywode (1996), Legal Form. Growth and Exit of West German Firms, Londres, CEPR, Discussion Paper, n ${ }^{\circ} 1401$.

Hull, C.J. et B. HJERN (1987), Helping Small Firms Grow. An Implementation Approach, Londres, Croom Helm.

ISTAT (2000), Rapporto sull' Italia, Bologna, Il Mulino.

Johnson, J., J. BALdwin et C. Hinchley (1997), Successful Entrants : Creating the Capacity for Survival and Growth, Statistique Canada, Ministère de l'Industrie.

Karlsson, C., B. Johannisson et D. Storey (dir.) (1993), Small Business Dynamics. International, National and Regional Perspectives, Londres, Routledge.

KLOFSTEN, M., D. JONES-EvAns et P. Lindell (1997), « Growth factors in technology-based spin-offs : a Swedish study », Piccola Impresa - Small Business, n 1, p. 13-37.

LEWIS, J., J. STANWORTH et A. GiBB (dir.) (1984), Success and Failure in Small Business, Aldershot, Gower.

MARCHINI, I. (1995a), a cura di, Il governo della piccola impresa. Le basi della conoscenza, vol. I, Genova, Ins-Edit.

MARChINI, I. (1995b), a cura di, Il governo della piccola impresa. La gestione strategica, vol. II, Genova, Ins-Edit.

Revue internationale P.M.E., vol. 14, $\mathrm{n}^{\text {os }} 3-4,2001$ 
MINISTRY OF ECONOMIC AFFAIRS - MEA (1998), Entrepreneurship in the Netherlands. New Firms : The Key to Competitiveness and Growth, Den Haag, SME Policy Unit.

MINISTRY OF ECONOMIC AFFAIRS - MEA (1999a), Entrepreneurship in the Netherlands. Ambitious Entrepreneurs : The Driving Force for the Next Millennium, Den Haag, SME Policy Unit.

Ministry OF ECONOMIC AFFAIRS - MEA (1999b), High-growth Companies in the Netherlands, Den Haag, SME Policy Unit.

Mussati, G. (1990), a cura di, Alle origini dell'imprenditorialità. La nascita di nuove imprese : analisi teorica e verifiche empiriche, Milano, Etas Libri.

Mutinelli, M. (1997), Le piccole multinazionali italiane, Rome, Edizioni Seat.

ODAKA, K. et M. SAWAi (dir.) (1999), Small Firms, Large Concerns. The Development of Small Business in Comparative Perspective, Oxford, Oxford University Press.

OCDE (1997), Small Business, Job Creation and Growth. Facts, Obstacles and Best Practices, Paris, OCDE Publications.

OCDE (2000), OECD Small and Medium Enterprise Outlook, Paris, OCDE Publications.

Pencarelli, T. (1995), Piccola impresa, alleanze strategiche e integrazione europea, Genova, Ins-Edit.

PRENCIPE, A. (1997), «The role of business innovation centers for regional development: the case of Italy », Piccola Impresa - Small Business, n 1, p. 97-113.

PRETI, P. (1991), L'organizzazione della piccola impresa : nascita e sviluppo delle imprese minori, Milan, Egea.

PRETI, P. (2001), Da un uomo solo al comando al gruppo dirigente : temi di organizzazione aziendale per la piccola impresa, Milan, Egea.

QuAdrio Curzio, A. et M. Fortis (2000), a cura di, Il made in Italy oltre il 2000. Innovazione e comunità locali, Bologna, Il Mulino.

RYCROFT, R.W. et D.E. KASH (1999), The Complexity Challenge. Technological Innovation for the 21th Century, Londres, Pinter.

RUllani, E. et A. RomANo (dir.) (1998), Il postfordismo. Idee per il capitalismo prossimo venturo, Milan, Etas Libri.

SCOTT, M. et R. BRUCE (1987), «Five stages of growth in small business », Long Range Planning, $\mathrm{n}^{\mathrm{o}} 3$.

SolinAS, G. (1996), I processi di formazione, la crescita e la sopravvivenza delle piccole imprese, Milan, Franco Angeli.

Stanworth, J., S. Blythe, B. Granger et C. Stanworth (1989), «Who becomes an entrepreneur? », Piccola Impresa - Small Business, no 2, p. 3-19.

StOREY, D.J. (1995), Understanding the Small Business Sector, Londres, Routledge.

STOREY, D.J. (1997), The Ten Percenters, Londres, Deloitte \& Touche.

TARANTO, S. (1990), Medie e piccole imprese nel nuovo scenario degli anni novanta, Rome, SIPI.

Veltz, P. (1996), Mondialisation, villes et territoires. L'économie d'archipel, Paris, Presses universitaires de France.

Revue internationale P.M.E., vol. 14, $\mathrm{n}^{\text {os }} 3-4,2001$ 